\title{
Soft robotics: Biological inspiration, state of the art, and future research
}

\author{
Deepak Trivedi, ${ }^{a}$ Christopher D. Rahn, ${ }^{\mathrm{a} *}$ William M. Kier ${ }^{\mathrm{b}}$ and Ian D. Walker ${ }^{\mathrm{c}}$ \\ ${ }^{a}$ Department of Mechanical and Nuclear Engineering, The Pennsylvania State University, University Park, PA 16802, USA; ${ }^{b}$ Department \\ of Biology, The University of North Carolina at Chapel Hill, Chapel Hill, NC 27599, USA; ${ }^{c}$ Department of Electrical and Computer \\ Engineering, Clemson University, Clemson, SC 29634, USA
}

(Received 4 March 2008; final version received 16 October 2008)

\begin{abstract}
Traditional robots have rigid underlying structures that limit their ability to interact with their environment. For example, conventional robot manipulators have rigid links and can manipulate objects using only their specialised end effectors. These robots often encounter difficulties operating in unstructured and highly congested environments. A variety of animals and plants exhibit complex movement with soft structures devoid of rigid components. Muscular hydrostats (e.g. octopus arms and elephant trunks) are almost entirely composed of muscle and connective tissue and plant cells can change shape when pressurised by osmosis. Researchers have been inspired by biology to design and build soft robots. With a soft structure and redundant degrees of freedom, these robots can be used for delicate tasks in cluttered and/or unstructured environments. This paper discusses the novel capabilities of soft robots, describes examples from nature that provide biological inspiration, surveys the state of the art and outlines existing challenges in soft robot design, modelling, fabrication and control.
\end{abstract}

Keywords: soft robots; muscular hydrostats; electroactive polymers; pneumatic air muscles

\section{Introduction}

Robots can be classified as hard or soft on the basis of the compliance of their underlying materials, as shown in Figure 1. A soft robot is inherently compliant and exhibits large strains in normal operation. Over the last 10 years, researchers have developed soft robots that provide new capabilities relative to traditional, hard robots. Table 1 compares the characteristics and capabilities of soft and hard robots. The most commonly used hard robots are kinematically nonredundant. These robots are typically used in well-defined environments in which they repetitively perform a prescribed motion with great precision. This capability is exploited in many successful applications, primarily in manufacturing. These robots are designed to be stiff so that vibration and deformation of the structure and drivetrain do not reduce the accuracy of movement. In general, hard robots have multiple flexible joints connected by stiff links. Each joint is flexible in one rotary or translational direction to provide a degree of freedom (df) of robot motion. The combined motion of all the df sweeps out the workspace or the locus of points that the tip position can attain. Hard robots are hyperredundant when the number of joints is very large. Robots made of certain hard materials such as shape memory alloys (SMAS) can also be designed to have continuous deformation and thus infinite df. Hyperredundant robots have the potential to work in unstructured environments and provide high dexterity.
Soft robots and traditional hard robots use different mechanisms to enable dexterous mobility (see Figure 2(a)). Soft robots have distributed deformation with theoretically an infinite number of $\mathrm{df}$. This leads to a hyper-redundant configuration space wherein the robot tip can attain every point in the three-dimensional workspace with an infinite number of robot shapes or configurations. Soft robots have an additional advantage over hard hyper-redundant robots in that they generate little resistance to compressive forces and therefore can conform to obstacles. Therefore, they can carry soft and fragile payloads without causing damage. Using large strain deformation, they can squeeze through openings smaller than their nominal dimensions. This makes them ideal for applications such as personal robots that interact with people without causing injury, service and painting robots that need high dexterity to reach confined spaces, medical robots, especially for use in surgery, and defence and rescue robots that operate in unstructured environments.

Soft robots differ from hard, hyper-redundant (e.g. serpentine and continuum) robots. Hirose (1993) reviewed the applications, kinematics and design of serpentine robots. Robinson and Davies(1999) presented a short review of continuum robots, distinguishing them from discrete (nonredundant) and serpentine (hyper-redundant) robots. They further classified continuum robots as intrinsic, extrinsic or hybrid on the basis of the method and location of

\footnotetext{
*Corresponding author. Email: cdrahn@psu.edu
} 
Table 1. Characteristics of different types of hard (first three columns) and soft robots.

\begin{tabular}{|c|c|c|c|c|}
\hline & Rigid & Discrete hyperredundant & Hard continuum & Soft \\
\hline \multicolumn{5}{|l|}{ Properties } \\
\hline $\mathrm{df}$ & Few & Large & Infinite & Infinite \\
\hline Actuators & Few, discrete & Many, discrete & Continuous & Continuous \\
\hline Material strain & None & None & Small & Large \\
\hline Materials & Metals, plastics & Metals, plastics & Shape memory alloy & $\begin{array}{l}\text { Rubber, } \\
\text { electroactive polymer }\end{array}$ \\
\hline \multicolumn{5}{|l|}{ Capabilities } \\
\hline Accuracy & Very high & High & High & Low \\
\hline Load capacity & High & Lower & Lower & Lowest \\
\hline Safety & Dangerous & Dangerous & Dangerous & Safe \\
\hline Dexterity & Low & High & High & High \\
\hline Working environment & Structured only & Structured and unstructured & Structured and unstructured & $\begin{array}{r}\text { Structured and } \\
\text { unstructured }\end{array}$ \\
\hline Manipulable objects & Fixed sized & Variable size & Variable size & Variablesize \\
\hline Conformability to obstacles & None & Good & Fair & Highest \\
\hline \multicolumn{5}{|c|}{ Design } \\
\hline Controllability & Easy & Medium & Difficult & Difficult \\
\hline Path planning & Easy & Harder & Difficult & Difficult \\
\hline Position Sensing & Easy & Harder & Difficult & Difficult \\
\hline Inspiration & Mammalian limbs & Snakes, fish & & Muscular hydrostats \\
\hline
\end{tabular}

mechanical actuation. Simaan et al. (2004) presented an overview of snake-like active bending devices used for minimally invasive surgery (MIS) of the throat.

The finite, controllable df of a soft robot are dictated by the actuators. Rigid-linked robots have an actuator, typically an electric motor, for every joint. The actuators of soft robots are typically integrated into and distributed throughout the structure. Often, the actuators make up most of the structure. This dual actuator/structure functionality prevents the use of many traditional hard actuators such as electric motors in soft robots. The deformation resulting from activation of an actuator is defined by the actuation mechanism and strain and the actuator size, shape and location in the structure. Soft robots fall into a class of systems that are termed 'underactuated' because, unlike hard robots, there is not an actuator for every $\mathrm{df}$. Other $\mathrm{df}$ may be influenced by the actuators, but many $\mathrm{df}$ are not controllable.

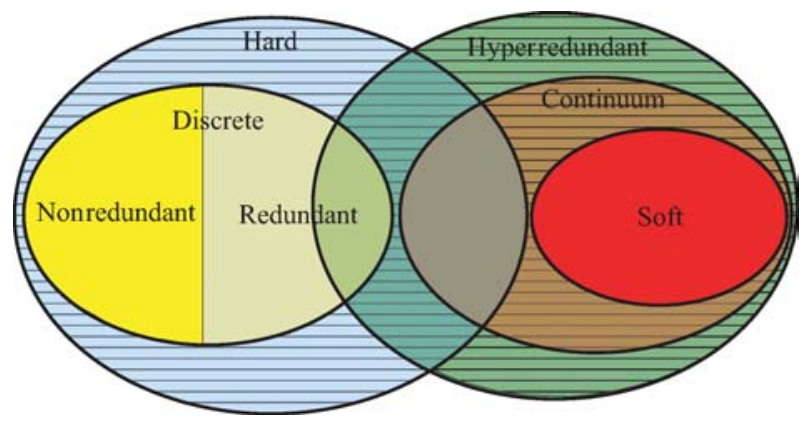

Figure 1. Classification of robots based on materials and degrees of freedom. Hatched area represents empty set.
Sensing and controlling the shape of a soft robot are challenging. Their structure is a continuum, so exact measurement of the shape and tip position is difficult. Deciding what to measure and how to use the measurements to control mobility is challenging. Hard robots measure the position of each joint with a high-resolution encoder as shown in Figure 2(b). Assuming a rigid robot, the joint positions can be processed by the forward kinematics to accurately determine the shape and tip position of the robot. Similarly, the inverse kinematics can be used to determine the joint positions that provide a desired tip position. The joint positions

(a)

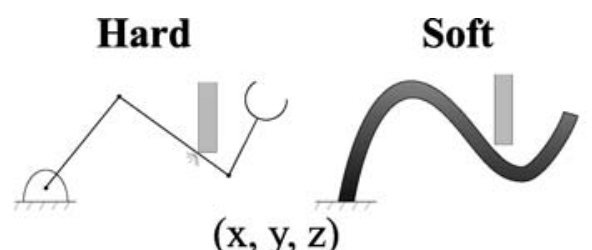

(b)

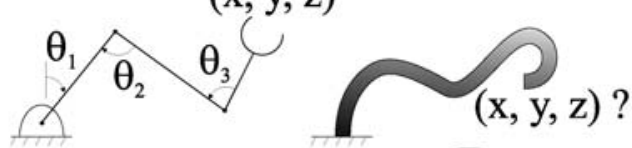

(c)

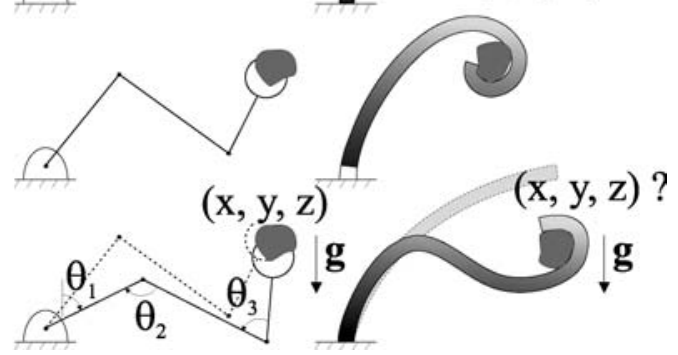

Figure 2. Capabilities of hard and soft robots: (a) dexterity, (b) position sensing, (c) manipulation and (d) loading. 


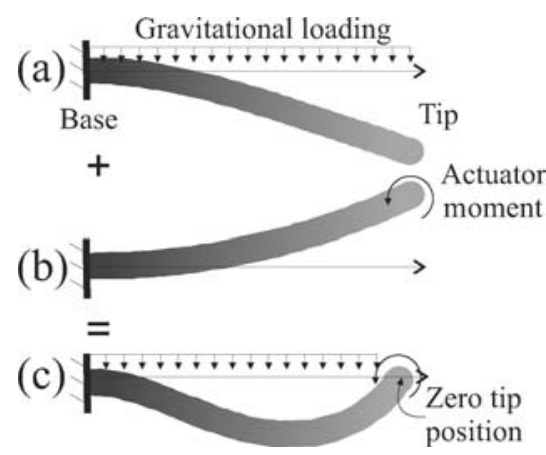

Figure 3. Gravitational loading on a soft robot manipulator causes downward deflection (a), actuators bend the structure upward (b) and net displacement has zero tip position but nonzero shape (c).

measured by the encoders are compared with the desired positions calculated from the inverse kinematics and the actuators then servo the errors to zero. This servo action is typically quite fast and forces the joints to precisely track their desired positions.

Soft robots interact with the environment differently from their hard counterparts. The environment applies loads to the structure either through distributed loading (e.g. gravity) or by contact. In a rigid-linked robot, Figure 2(c) shows that loading causes the soft joints to change position whereas the rigid links remain straight. The encoders measure the position change, and the controller can either compensate for the loading or understand that the robot has contacted the surroundings. In either case, the shape and the tip position can be exactly determined. Gravity and contact loading cause continuous deformation in a soft robot that may not be observable or controllable from the limited sensors or actuators, respectively.

Contact and conformation with the surrounding environment play an important role in the mobility of soft robots. Soft robot arms, for example, use whole arm manipulation to grasp and handle objects of varying size as shown in Figure 2(d). The arm wraps around the object and a tight grasp and a high-friction contact enable the arm to lift the object. Hard robot arms grasp and handle objects with a specialised end effector that is typically designed for a specific size and type of object. Soft robots can locomote by using a variety of gaits, with a large portion of their structure in ground contact at any instant in time. Hard robots use separate legs, tracks and wheels to contact the ground and enable locomotion.

Figure 3 shows how a soft robot arm deforms under combined gravitational loading and actuation. Hard robots can servo the arm to any shape if the links are sufficiently stiff and the load is sufficiently low. The actuators on a soft robot arm often apply a moment or a torque at the tip of the arm. For small displacements, this tip moment causes the arm to bend upward with a quadratic shape. In a gravi- tational environment, self-weight bends the arm downward with a cubic shape. The tip moment can be adjusted to lift the tip to the horizontal, position but the arm will have a non-zero shape associated with the difference between the quadratic and cubic shapes (Pritts 2005). Similarly, if a sensor is employed that measures the moment at the base of this soft robot arm, one cannot differentiate between a point load at the tip and a distributed load. These loadings, however, produce markedly different arm shapes.

This paper is the first review to focus exclusively on soft robots. The state of the art is discussed and research challenges are presented. Biological inspiration in the design and control of soft robots is presented and the complexities of fabrication, modelling and path planning are introduced.

\section{Biological inspiration}

There are many examples in nature of mobile structures made from soft materials. Muscular hydrostats such as elephant trunks, mammal and lizard tongues and octopus arms are soft structures that can bend, extend and twist. Fibre reinforcement in soft plant cell walls enables the cell to change shape when pressurised. Mimicry of these complex structures is neither necessary nor practical to the development of soft robots. Fundamental understanding of the morphology and functionality of soft structures in nature, however, increases insight and can lead to new design concepts in soft robotics. The natural world demonstrates the potential capabilities of soft robots.

\subsection{Hydrostatic skeletons and muscular hydrostats}

Animals such as worms and sea anemones lack the rigid jointed skeletons that are found, for instance, in the vertebrates (e.g. mammals, birds and reptiles) and arthropods (e.g. insects and crabs). Instead, these soft-bodied animals depend on a 'hydrostatic skeleton' for support (Chapman 1958, 1975; Clark 1964; Gutmann 1981) . Hydrostatic skeletons are typically cylindrical, fluid-filled cavities surrounded by a muscular wall that is reinforced with connective tissue fibres. The fluid is usually a liquid (essentially water) and thus resists significant volume change. Thus, if muscle fibres in the wall contract to decrease one of the dimensions, another dimension must increase. By arranging the musculature so that all dimensions can be actively controlled, a diverse array of movements and shape change can be produced. Force transmission is thus provided not by rigid links but instead by pressure in the enclosed fluid. This simple principle serves as the basis of support and movement in a diverse group of soft-bodied animals. Hydrostatic skeletal support may also be important in organisms that typically rely on rigid skeletons. For example, crabs rely on hydrostatic skeletal support after they have shed their exoskeleton during molting and before the newly formed 

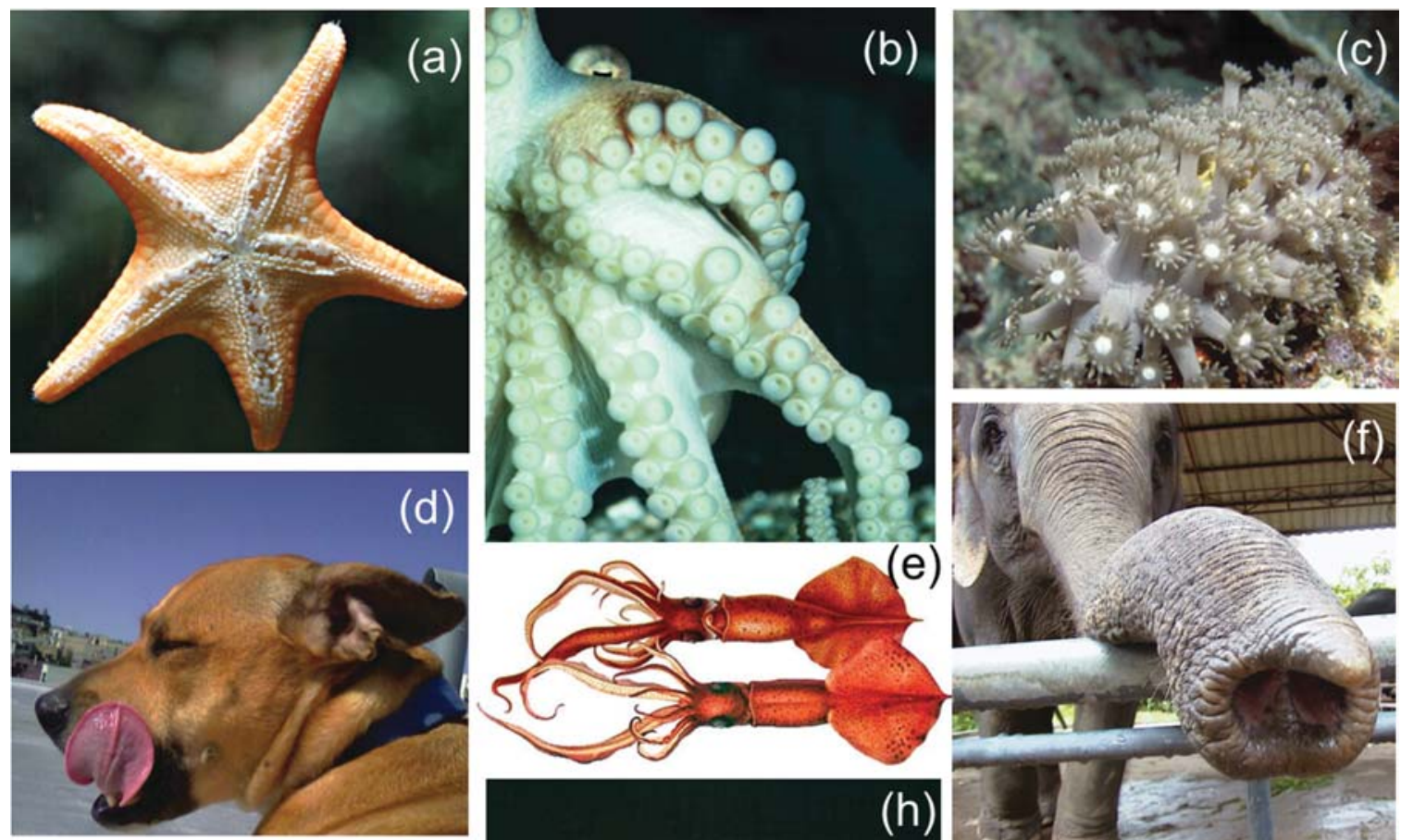

(d)
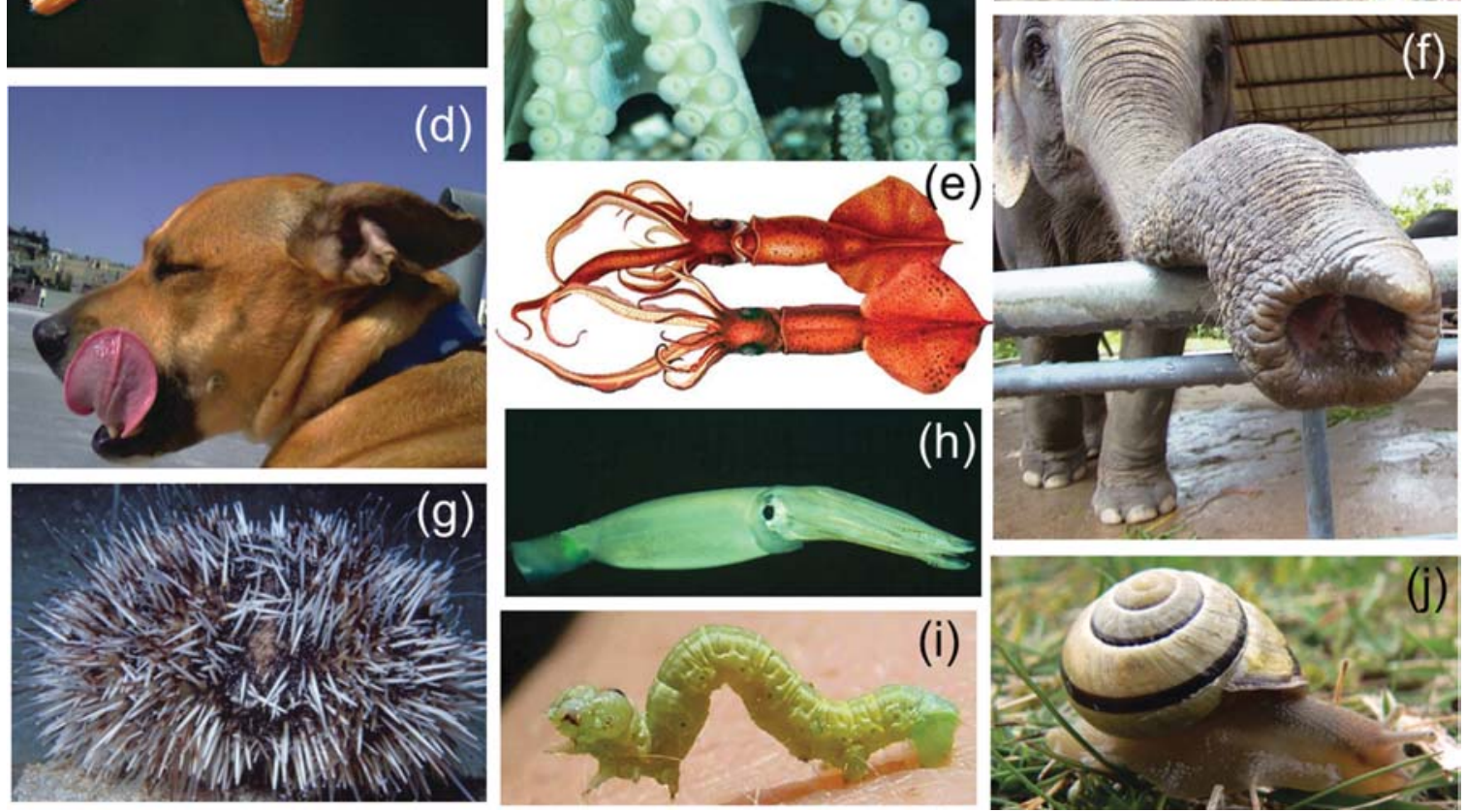

Figure 4. Examples of hydroskeletons and muscular hydrostats: (a) tube feet in starfish, (b) octopus arms, (c) colonial anemone, (d) mammalian tongue, (e) squid, (f) elephant trunk, (g) echinoid, (h) Illex illecebrosus, (i) inchworm, and (j) snail feet.

cuticle has hardened (Taylor and Kier 2003, 2006) and hydrostatic pressure under the skin of sharks may provide a means of transmitting force to the tail (Wainwright et al. 1978).

In addition to large fluid-filled spaces and muscle fibres arranged in multiple orientations, the walls of most hydrostatic skeletons are reinforced with connective tissue fibres (most commonly the protein collagen) arranged as continuous parallel sheets of fibres that wrap the animal in both left- and right-handed helical arrays. Such 'crossed-fibre helical connective tissue arrays' provide reinforcement for the walls and allow both smooth bending and change in length (Wain-wright 1982, 1988). The fibre angle, the angle that the fibres make with the long axis, has been shown to control and limit shape change in a variety of wormlike animals (Harris and Crofton, 1957; Clark and Cowey 1958) and hydraulic structures, such as the tube feet of echinoderms (starfish, sea urchins, brittlestars, sea cucumbers) (McCurley and Kier 1995).
Kier and Smith (1985) introduced the term 'muscular hydrostat' to describe a group of soft animal structures that lack the large fluid-filled cavities that characterise the hydrostatic skeletal support systems of other soft-bodied animals. Examples of muscular hydrostats include the arms of octopuses, the arms and tentacles of squid, many tongues, the elephant trunk and a variety of invertebrate structures (see Figure 4). These structures are typically capable of diverse and complex movement and are unusual because the musculature generates both the force for movement and provides skeletal support. Support and movement are achieved in a similar way to conventional hydrostatic skeletons by exploiting the near incompressibility of muscle at physiological pressures and arranging the musculature to control all three dimensions. The morphology and biomechanics of several muscular hydrostats have been examined including squid arms and tentacles (Kier and Smith 1985), squid and cuttlefish fins (Kier 1989), chambered nautilus tentacles (Kier 1987), octopus suckers (Kier and Smith 
1990), chameleon tongue (Herrel et al. 2001), microhylid frog tongue (Meyers et al. 1999), human tongue (Napadow et al. 2002) and African pig-nosed frog tongue (Nishikawa et al. 1999).

Muscles and reinforcing connective tissue play an important role in the functionality of muscular hydrostats. Animal muscle is particularly well suited to soft actuation. Meijer et al. (2003) summarised the range of performance metrics of muscle including the maximum force production at constant length, length dependence of force production, the rate at which force can be generated and velocity dependence of force production. Muscles, while all being contractile, have wide variability in characteristics among different species and even among different muscles in the same animal (Hunter and Lafontaine 1992). For instance, the extensor musculature of the squid tentacle contracts at a peak velocity of approximately 15 lengths per second and shows a peak stress of approximately $130 \mathrm{mN} \mathrm{mm}^{-2}$, whereas the analogous musculature in the arms of squid (responsible for support of the arms) contracts at only 1.5 lengths per second but shows a peak stress of approximately $470 \mathrm{mN} \mathrm{mm}^{-2}$ (Kier and Thompson 2003; Kierand and Curtin 2002). And although the extensor musculature of the tentacles operates over a range of strain of less than $30 \%$, the retractor musculature of the tentacles operates over a range of strain of greater than $80 \%$ (van Leeuwen and Kier 1997). As there are limited strain amplification mechanisms in soft structures, high strain is often required for high mobility. Inactivated muscle can be easily extensible and allow large deformation with limited stress. When activated, however, the stress can be large to enable the structure to do significant work on the environment. This variable stress capability of animal muscle gives muscular hydrostats unique dexterity and load-bearing capabilities.

As an example of a sophisticated muscular hydrostat, Figure 5 shows the morphology of an octopus arm (Kier and Stella 2007). Layers of muscle with different orientations wrap around the central nerve cord. Fibres of the core of transverse musculature are oriented in the radial direction and interleave with the next two layers of oblique musculature (OM) and longitudinal musculature (LM), which helically wound around the arm and aligned along the axis of the arm, respectively. Two layers of OM separated by a layer of LM surround the arm just under the external skin. The oblique muscle fibre layers are wound in both the clockwise $(\mathrm{CW})$ and counter-clockwise $(\mathrm{CCW})$ directions. The muscle layers are integrated with both discrete layers of connective tissue and networks of connective tissue fibres in the musculature. Contraction of the longitudinal muscle fibres causes the arm to shorten. Contraction of the transverse muscle fibres acts antagonistically to the longitudinal muscle fibres to cause arm extension. Simultaneous contraction of the transverse and longitudinal muscle fibres increases the flexural stiffness of the arm, allowing it to bear loads. If the longitudinal muscle fibres are not

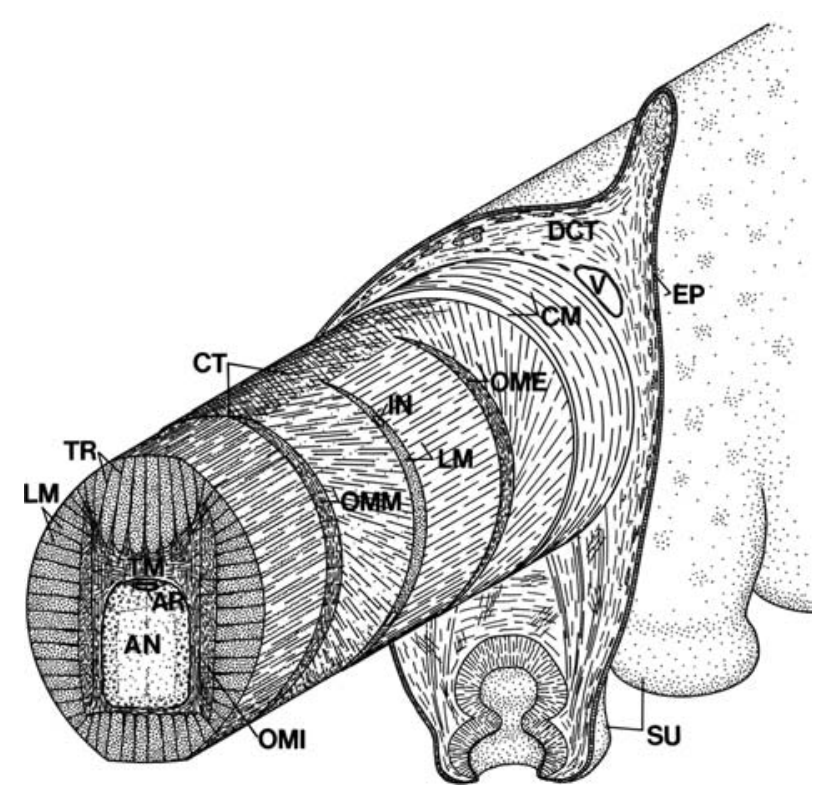

Figure 5. Diagram of the arm of Octopus showing threedimensional arrangement of muscle fibres and connective tissue fibres. AN, axial nerve cord; AR, artery; CM, circumferential muscle layer; CT, connective tissue; DCT, dermal connective tissue; EP, epidermis; IN, intramuscular nerve; LM, longitudinal muscle fibres; OME; external oblique muscle layer; OMI, internal oblique muscle layer; OMM, median oblique muscle layer; SU, sucker; TM, transverse muscle fibres; TR, trabeculae; and V, vein.

uniformly contracted around the circumference, the arm bends in the softest direction. Thus, activating the longitudinal and transverse muscle fibres along the length and around the circumference of the arm causes the arm to bend in complex shapes. Activation of the $\mathrm{CW}$ and $\mathrm{CCW}$ oblique muscle fibre layers twists the arm in the $\mathrm{CCW}$ and $\mathrm{CW}$ directions, respectively. This complex structure of soft active material and connective tissue can produce large and convoluted extension, bending and twisting motions.

\subsection{Soft active plant structures}

In many plants, the soft cell walls are reinforced by stiff fibrillar networks. A variety of osmotic processes pressurise the cell in response to different stimuli (e.g. light or pressure), leading to plant movement. The fibres are oriented to ensure that the cell deforms in a specific direction when pressurised. The guard cell shown in Figure 6, for example, controls aspiration in plant leaves, thereby limiting water loss (Hetherington 2001). The microfibrils are wound around the circumference of the cell at an angle to the curved longitudinal axis. When pressurised by osmosis, the guard cells deform to open the stoma and allow gas exchange to take place. If large numbers of cells are aligned in a plant structure, they can cause macroscopic changes in shape. This mechanism is responsible for phototropism or sun tracking (Takagi 2002) and the large and fast 


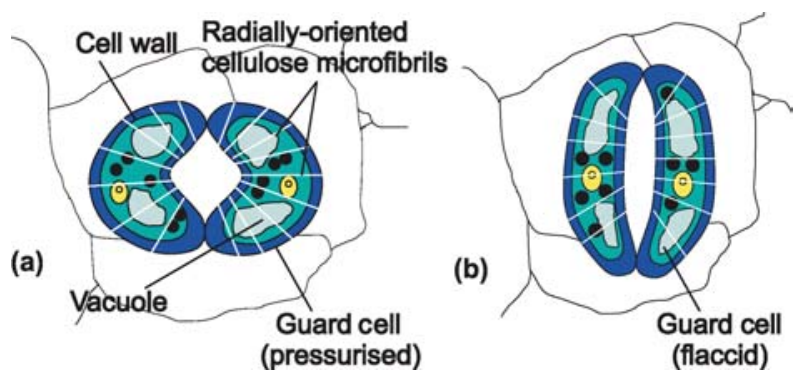

Figure 6. A schematic of open (a) and closed (b) stomata in plant cells caused by osmotic pressurisation.

deformation of the Venus fly trap and Mimosa plants (Hodick and Sievers 1989, Kanzawa et al. 2006).

\section{State of the art in soft robotics}

Inspired by the outstanding capabilities of soft animal and plant structures researchers have developed hard robots that mimic soft structures and soft robots that use electroactive polymer (EAP) and pneumatic artificial muscle (PAM) actuators. Most soft robots developed so far are based on these two technologies, and few examples of other kinds of soft robots exist. The octopus arm and guard cell indicate the potential for soft mobile structures, but significant challenges remain in the development of soft robots and specifically the areas of active materials, electromechanical design, modelling for optimisation and control, and fabrication.This section describes some of the most interesting examples of soft terrestrial and aquatic robots and manipulators that have been built and experimentally tested in the last 20 years.

\subsection{Hard robots with soft capabilities}

Robotics researchers have developed a variety of terrestrial (wheeled, tracked, crawling and legged) and aquatic (swimming) robots and manipulators that employ primarily rigid materials but achieve flexibility that is reminiscent of biological structures. Notable examples of hard robots that are inspired by soft structures found in nature include crawling and swimming robots and trunk-like manipulators. Crawling robots use undulatory locomotion based on the coupling between the robot deformations and the ground (Ostrowski and Burdick 1998). Examples of crawling robots include snake-like climbing robots (Wright et al. 2007) and snake robots (Miller 2007). Swimming robots that mimic the continuous motion of fish using a rigid linkage include the thunniform robots called RoboTuna I and II (RoboTuna 2001), the AQUA Project (Georgiades et al. 2004), Essex robot fish (Liu et al. 2006), a micro underwater vehicle (Deng and Avadhanuala 2005), an amphibious snake-like robot (Crespi and Ijspeert 2006) and the Boxybot fish that can dive, move forwards and backwards, swim on its side and spin (Lachat et al. 2006). These robots do not provide the more efficient and noiseless continuous motion of a soft, flexible swimming body (Guo et al. 2003). There are many legged robots with rigid structures that use electric/magnetic, piezoactive or thermal (SMA) actuation (Daugela et al. 1995). Sugiyama and Hirai(2006) developed a spherical robot that can crawl and jump by using deformation of its SMA body. Trimmer (2006a) built a robotic climbing machine ("Softbot") based on the biomechanics and neuromechanics of the caterpillar Manduca sexta. The body of this robot is made of highly elastic silicone rubber and is actuated by using springs made of SMA, bonded to the inside of the body wall. The robot is continuously deformable and capable of collapsing into a small volume. Examples such as these show that it is possible to achieve most capabilities of soft robots even when the underlying actuating materials are hard.

Trunk-like manipulators have also been built by using rigid structures and electric motors with cable tendons for actuation. Cieslak and Morecki (1999) developed an elastic manipulator by using cable tendon actuators. Hannan and Walker (2001) developed a four-section 'elephant trunk' manipulator with sections actuated by a hybrid cable and spring servo system (see Figure7). OCRobotics builds trunk-like commercial robots called 'snake-arm robots' (Buckingham and Graham 2003) also using cable tendon actuators with alternating rigid and soft disks to form a bendable backbone. Simaan (2004) and Wei et al. (2006) developed a manipulator for MIS of the throat, composed of a base disk, an end disk, several spacer disks, four superelastic backbone tubes and three push-pull actuators. Sears and Dupont (2006) and Webster et al. (2006) proposed MIS tools that derive bending actuation by relative rotation and extension of curved concentric tubes.

\subsection{Soft EAP robots}

EAPs (Bar-Cohen 2000, Samatham et al. 2007) have many characteristics, including low weight, fracture tolerance, pliability and relatively large actuation strain that make them especially suitable for soft robots (Meijer et al. 2001). EAPs can be broadly classified into electronic EAPs (dielectric elastomers, electrostrictive graft elastomers, electrostrictive paper, electroviscoelastic polymers ferroelectric polymers and liquid crystal elastomers) and ionic EAPs (carbon nanotubes, conductive polymers, electrorheological fluids, ionic polymer gels and ionic polymer metallic composites) (Meijer et al. 2003). In general, ionic EAPs operate at low voltage but require constant hydration and produce low stress, limiting their applications. On the other hand, electronic EAPs produce relatively large strains, respond quickly and are relatively efficient, but often require high actuation voltages (Meijer et al. 2003).

Pelrine et al. (2002) concluded that dielectric elastomers (Kornbluh et al. 1998; Zang et al. 2005) are closest to animal 


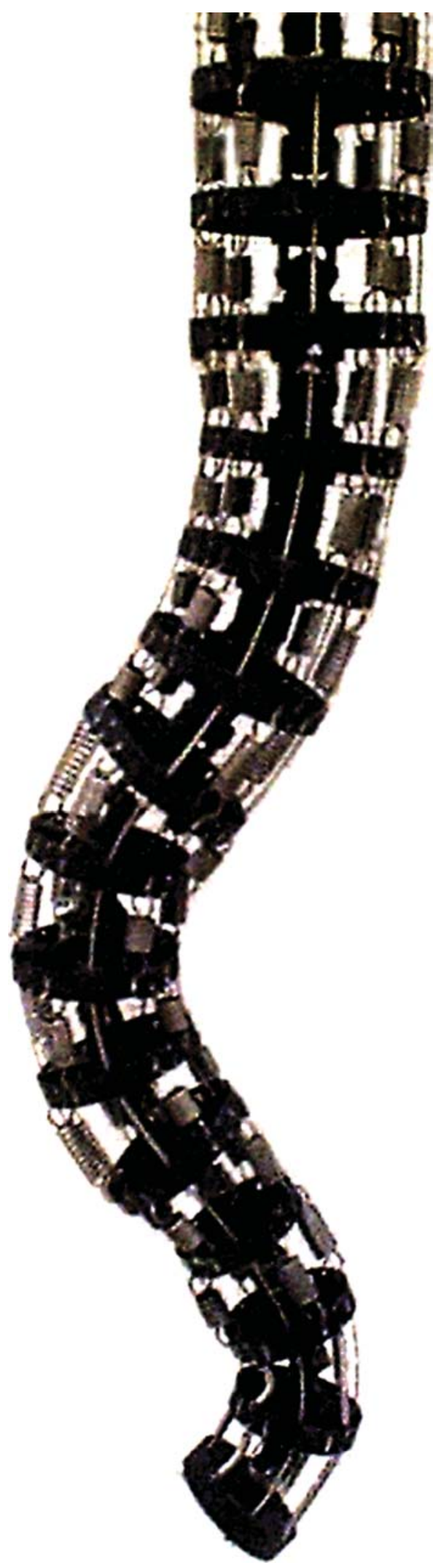

Figure 7. Elephant trunk manipulator, an example of hard hyperredundant robot. muscles based on criteria of strain, actuation pressure, density, efficiency and speed. Interesting applications of dielectric elastomers in soft robotics include a lightweight six-legged robot (Eckerle et al. 2001), a series of legged robots called MERbots (Pei et al. 2004) that use multifunctional electroelastomer rolls and lightweight robots with actuators that mimic the longitudinal muscles of earthworms for locomotion (Jung et al. 2007). A hyper-redundant digital manipulator driven by embedded dielectric polymer actuators can perform precise discrete motions without the need for sensing and feedback control (Wingert et al. 2002).

Actuators developed by using conducting polymers (Cortés and Moreno 2003) have been used to build many biologically inspired robots. Nie et al. (2006), for example, developed a tortoise-like flexible microrobot that can crawl and swim underwater by using four legs actuated by ionic conducting polymer film (ICPF). Likewise, Guo et al. (2003) developed a 45-mm long fish-like underwater microrobot using ICPF actuators made from perfluorosulfonic acid polymer films that drive a pair of tails with fins. Speeds from 0 to more than $5 \mathrm{~mm} / \mathrm{s}$ can be obtained by the changing frequency and amplitude of the input voltage from 0.1 to $5 \mathrm{~Hz}$ and $0.5 \mathrm{~V}$ to $10 \mathrm{~V}$, respectively. Microgrippers (e.g. Alici and Huynh 2006, 2007) and other actuated devices have been fabricated by using conjugated polymers. Micromuscle AB (Micromuscle 2007) actuation devices based on conjugated polymers, including tubes for blood vessel sealers are commercially available.

Ionic polymer-metal composites are a popular material for use in soft robots (Shahinpoor et al.1998, Shahinpoor and Kim 2001; Nakabo et al. 2007). Successful applications include wormlike robots (Arena et al. 2006; Pak et al. 2006) that imitate the travelling wave observed in undulatory locomotion, an underwater propulsion robot that uses the IPMC as a fin to generate forward impelling force (Mojarrad and Shanhinpoor 1997), fish-like vehicles (Jalbert et al. 1995; Ijspeert and Kodjabachain 1999; Kerrebrock et al. 2001; Guo et al. 2003; Jung et al. 2003), a ciliary based eight-legged walking microrobot (Kim et al. 2003) and a multi-df micromanipulator (Tadokoro et al. 1999). IPMC actuators are susceptible to dehydration and degradation, however, control of these materials is complicated by the poor understanding of their chemical and physical structures (Bar-Cohen 2000).

EAP gels (Cortés and Moreno 2003) have been used to fabricate a hand with gel fingers (Shiga et al. 1989) and gel robots (Otake et al. 2002). The gel robots are made entirely of EAP gel that changes shape under spatially varying electric fields. Figure 8 shows a starfish gel robot that turns over application of electric fields. Tabata et al. (2001) used self-oscillating gel to develop a ciliary motion actuator. The dynamics of the actuator is similar to oscillatory (e.g. beating heart) biological phenomena. 

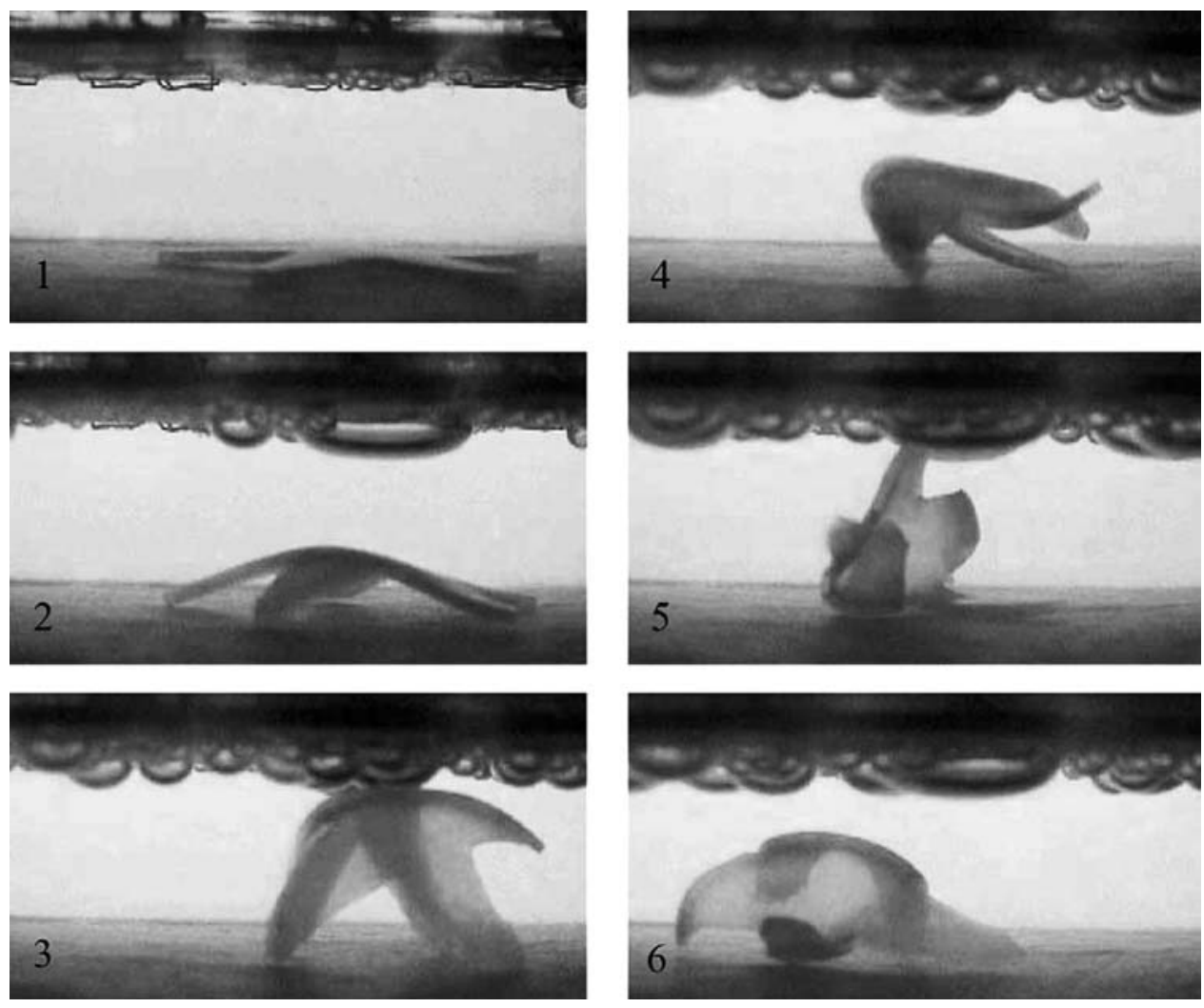

Figure 8. A starfish gel robot that turns over (Otake et al. 2002).

Soft actuators based on liquid crystal elastomers (O'Halloran and O'Malley 2001) and electrostrictive graft elastomers (Bar-Cohen 2000; Madden et al. 2004) have also been developed but not used in soft robotics.

EAPs suffer from several shortcomings that make their extensive use in soft robots difficult. Most ionic EAPs can work only in aqueous media (Bar- Cohen 2000), conjugated polymers and ionic polymer-metal composites have short lifecycles due to creep and material degradation (Shahinpoor et al. 1998), EAP gels require very high voltage for actuation (up to $150 \mathrm{MV} / \mathrm{m}$ ) (Cortés and Moreno 2003) and most actuators made of EAPs are not significantly scalable. In spite of these challenges, EAPs remain a popular material for soft robots. The reversibility of actuation and sensing capabilities makes it possible to combine structural, actuation and sensing functions in the same material, making EAPs attractive for biomimetic applications. The first actuators based on EAPs are now being commercialised.

\subsection{Soft PAM Robots}

Daerden and Lefeber (2002) reviewed PAMs, also known as rubbertuators or braided pneumatic actuators (Figure 9), and defined them as pneumatic actuators that consist of a thin, flexible, tubular membrane with fibre reinforcement.
Braided and netted PAMs have high and low-density fibre mesh, respectively. The fibres can be either embedded in the wall of the actuator or applied as a braided sleeve on the outside of the tube. The well-known McKibben muscles have braided, embedded fibres (Kluate et al. 1999). Several other designs for PAMs have been patented (Morin 1953; Yarlott 1972; Kukolj 1988; Paynter 1988a, 1988b). The maximum contraction strain is approximately $57 \%$.

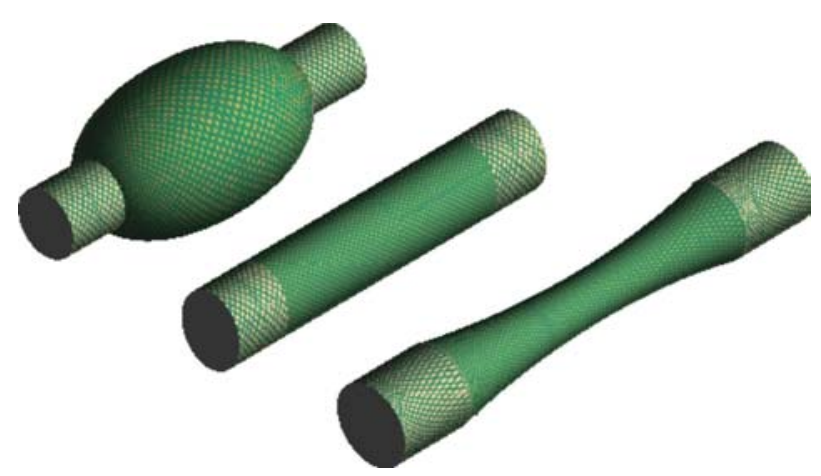

Figure 9. Pneumatic air muscle actuators with a mesh angle of less than $54^{\circ} 44^{\prime}$ contract on pressurisation (left), with a mesh angle of $54^{\circ} 44^{\prime}$ neither extend nor contract (middle), and of greater than $54^{\circ} 44^{\prime}$ extend on pressurisation (right). 

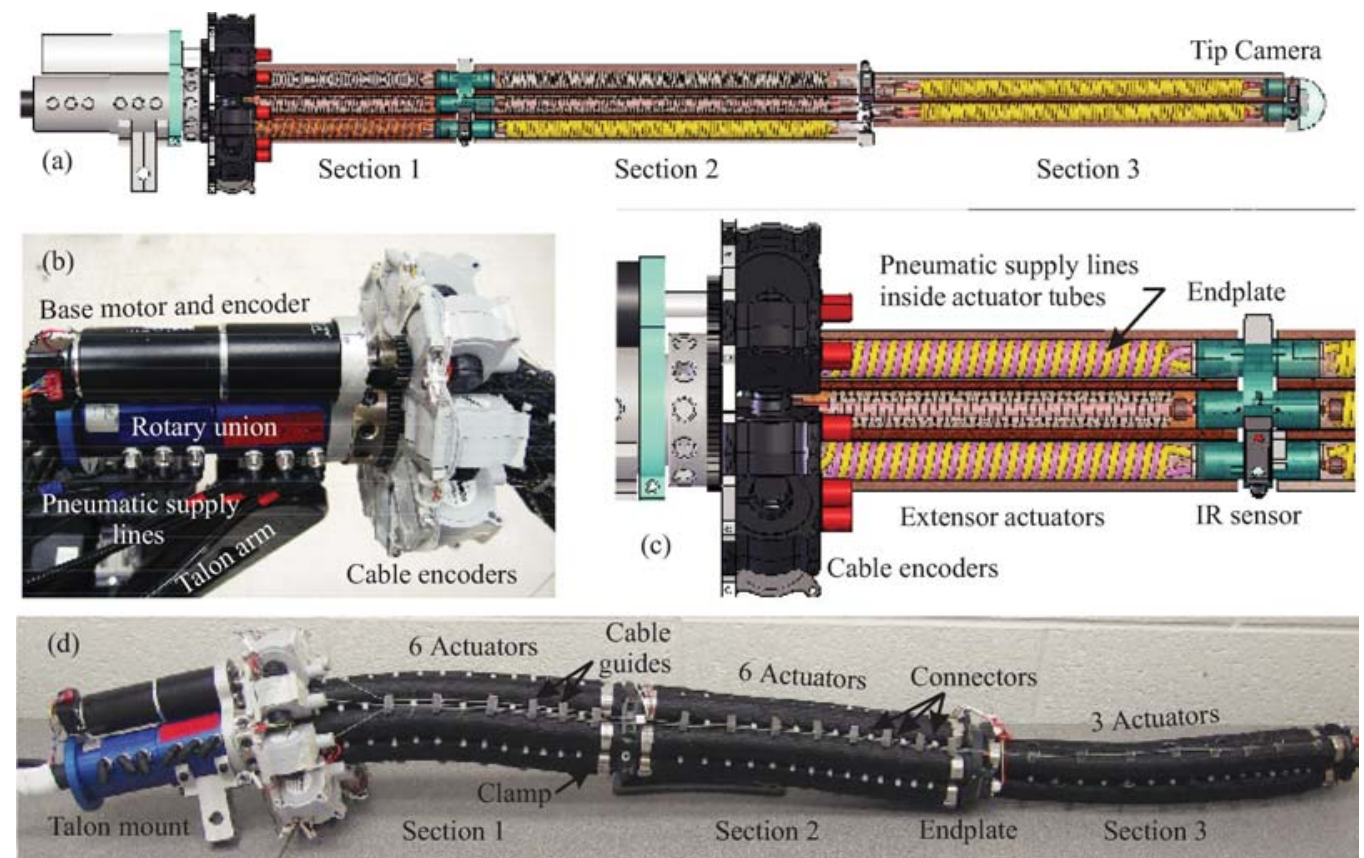

Figure 10. OctArm VI: (a) semi-transparent three-dimentional view of arm; (b) closed-up photograph of base; (c) closed-up, semitransparent view of first section and (d) photograph of complete arm (Dienno 2006).

The blocking force, which is the force required for constraining a fully energized actuator to zero displacement, however, can be extremely large due to the leveraging of the radial pressure through the braided fibres. As opposed to McKibben muscles, which contract, Pritts and Rahn (2004) introduced extensor actuators in which a large wind angle (above $54^{\circ} 44^{\prime}$, defined as the angle relative to the longitudinal axis) causes the actuator to extend upon pressurisation. Extensor actuators provide large extensional strain (200\% for a wind angle of $78^{\circ}$ ) but low extensional force. Additionally, they are prone to buckling instabilities under compressional axial loading.

PAMs have been used to make trunk-like soft robotic manipulators. Kinetic Sciences Inc. (Immega and Antonelli 1995), for example, developed a tentacular robot, powered by a hybrid system of pneumatic bellows and electric motors. It can extend, contract and bend in 6 df by using tendons threaded through cable guides. In contrast to bellows, Suzumori et al. (1991) developed a pneumatically and hydraulically driven flexible microactuator made of fibrereinforced rubber. The actuator has three internal chambers, each with a separate control valve. When the internal pressure in the three chambers is increased equally, the actuator stretches in the axial direction. When the pressure of only one of the chambers is increased, the actuator bends in a direction opposite to the pressurised chamber. Wilson et al. (1993) achieved bending without using separate chambers in a flexible four-section robotic arm manipulator by using orthotropic polyurethane tubes that bend when pressurised. Another actuation approach has been used in the Active Hose, an elephant trunk-type manipulator developed by Tsukagoshi et al. (2001), consisting of a spiral tube wound around the manipulator backbone like a coil. Mangan et al. (2002) used sequential actuation of a series of three McKibben actuators to develop an endoscope that can peristaltically locomote forwards and backwards in the gastrointestinal tract. Menciassi and Dario (2003) developed microendoscopy devices inspired by inchworm locomotion. These devices have two types of actuators - a clamper to adhere the device to the locomotion environment and an extensor to produce a positive displacement. The extensor consists of a pneumatic bellow, whereas the clampers are activated either by exploiting the reversible phase change of diethyl ether leading to pressurisation of an elastic membrane or by using an actuation mechanism based on IPMCs.

Pritts and Rahn (2004) developed a soft robot manipulator with two sections that each provide two axis bending and extension. Half of the actuators in each section are extensors and the other half are contractors. OctArm VI (Dienno 2006) is the latest in a series of soft robotic manipulators designeded by using PAM extensors. Figure 10(a) shows a partially transparent three-dimensional view of the entire arm with a photograph of one of the two fabricated arms shown below in Figure 10(d). In this manipulator, extensors are connected together in groups of three and six to create three independently actuated sections. The sections can bend around the two transverse axes and extend longitudinally 


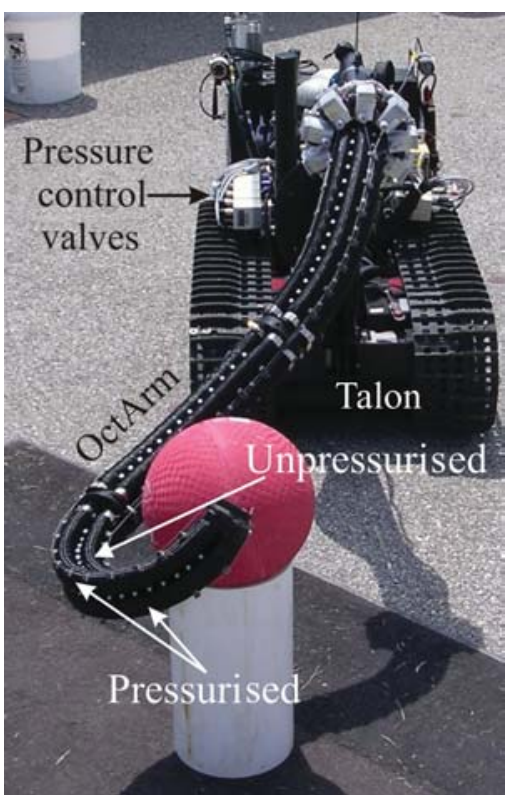

Figure 11. OctArm VI mounted on a mobile base (TALON), reaching around a ball.

when pressure is applied to each control channel and the corresponding extensors elongate. The three sections are connected in series and can be actuated independently through tubing that connects to pressure control valves on the mobile base, providing $9 \mathrm{dF}$. This provides the ability shown in Figure 11 to wrap the arm around objects.

Some PAM-based swimming robots have also been developed. Festo's Bionic Learning Network (Festo 2007) designs a swimming robot called Airacuda using pneumatic muscles made of rubber reinforced with aramid fibre. The robot has two muscles that are alternately pressurised to bend the tail and drive the fish forward. Suzumori et al. (2007) developed a soft-bodied manta swimming robot using an optimal pneumatic actuator cross-section and a new prototyping method. Chen et al. (1999) developed a metamorphic underwater vehicle that propels by changing shape through the inflation and deflation of water-filled latex balloons.

PAM-based actuators suffer from some shortcomings that restrict their applicability in soft robotics. They need a bulky air compressor for continuous operation, restricting mobility and making miniaturisation difficult. PAMs have a short fatigue life (10,000 cycles) (Samatham et al. 2007). Their advantages over EAP-based actuators is that they are chemically more stable and easy to work with. EAPand PAM-based actuators are suitable for different kinds of applications and both are likely to continue to be of interest to soft robotics researchers and developers. For instance, it is difficult scale up EAP-based robots and scale down PAM-based robots.

\subsection{Modelling}

The structural mechanics of soft actuators and manipulators, natural as well as artificial, is complicated owing to both material and geometric nonlinearities. Researchers developed biomechanical models that predict the behavior of several soft animal appendages. Skierczynski et al. (1996) developed a model of the hydrostatic leech skeleton to predict the shape of and internal pressure within the animal in response to motor neuron activity. Van Leeuwen and Kier (1997) presented a forward dynamics model of tentacle extension in squid to predict the changing geometry of the tentacle as well as the pressure, stress and kinetic energy distributions. Yekutieli et al. (2005) derived a two-dimensional dynamic multi-segment lumped parameter model of the octopus arm and use it to explore movement control strategies. Liang et al. (2006) presented a more sophisticated explicit finite-element simulation scheme for biological muscular hydrostats. Biomechanical models for tongue movements (Chiel et al. 1992; Napadow et al. 2002; Gerard 2003), caterpillar locomotion (Trimmer et al. 2006b) and elephant trunks (Wilson et al. 1991) have also been developed.

Modelling of soft robotic manipulators combines large deformation constitutive models of the active materials that form the manipulator with non-linear kinematics of the manipulator. Constitutive relations for active materials vary widely, but general models for the kinematics of soft robotics can be described by using, for example, spatially varying quaternions (Trivedi et al. 2007b). Soft robotic manipulators are kinematically similar to hyper-redundant manipulators with extremely large df, so models that approximate continuum manipulators by finite df hyper-redundant manipulators may be appropriate. In this approach, the accuracy and computational cost are proportional to the number of df (Chirikjian 1993). Many researchers (Chirikjian 1993, 1995, 1997) take the opposite approach by approximating the dynamics of hyper-redundant continuum manipulators. Mochiyama and Kobayashi $(1998,1999)$ and Mochiyama and Suzuki (2003) approximated the kinematics and dynamics of manipulators with hyper $\mathrm{df}$ using a continuum backbone curve and the Frenet-Serret formulas. For numerical simulation, however, they approximated the backbone curve by a serial chain of rigid bodies with a large number of df. Previous researchers account for the geometric nonlinearities by assuming fixed shapes for robot backbone curves. Hirose (1993) gave a planar model for snake robots based on a serpenoid backbone curve. Hannan and Walker (2000a) and Gravagne et al. (2003) assume that each section of an elephant trunk manipulator bends into a circular arc with constant curvature and an inextensible backbone (Hannan and Walker 2000b, 2003, Gravagne and Walker 2000b). Similarly, Nakabo et al. (2007) used the constant curvature assumption to study the kinematics of a snake-like swimming robot. This assumption makes it possible to formulate the forward manipulator kinematics 


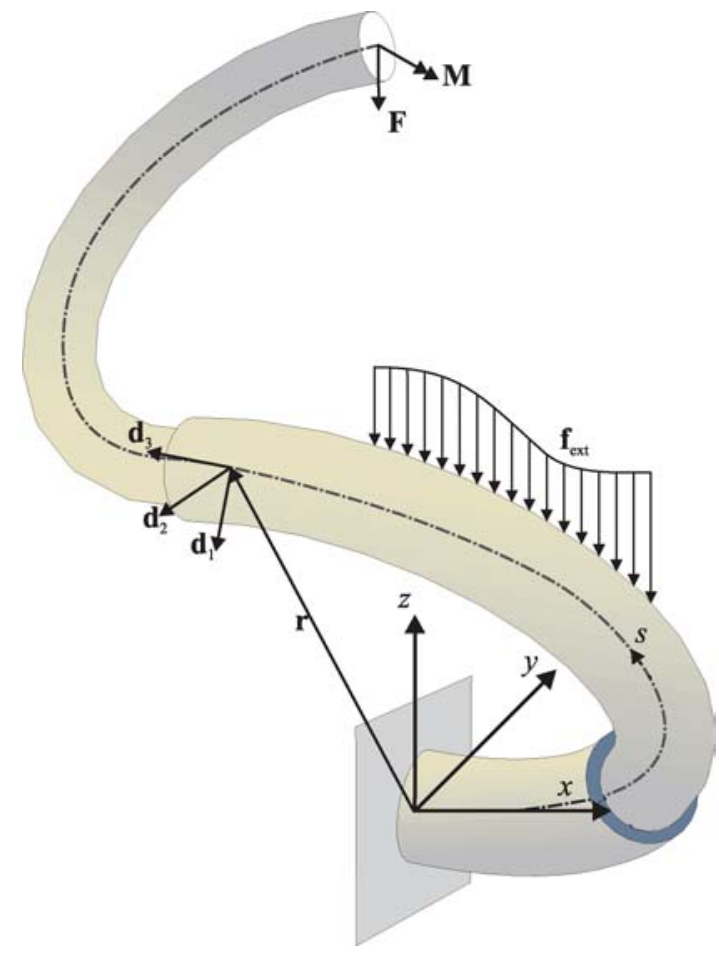

Figure 12. Soft robotic manipulator modelled by using the Cosserat rod approach, with the backbone position (r) and orientation $\left(\mathbf{d}_{1}, \mathbf{d}_{2}, \mathbf{d}_{3}\right)$ parametrised by a single variable $s$. The manipulator is acted upon by distributed force (f) and discrete forces $(\mathbf{F})$ and moments $(\mathbf{M})$.

that relate the position of the manipulator tip to the inputs by using a Denavit-Hartenberg formulation that fits a virtual rigid-link robot to the continuum backbone. Recent work by Jones and Walker (2006) extends this approach by removing the assumption of an inextensible backbone. These methods rely, however, on the assumption of constant curvature that is valid only when there are no external loads (including self-weight) on the manipulator. Self-weight and other loading can cause significant deviations from constant curvature, leading to large tip position error. Tatlicioglu et al. (2007) extended the model to include the effects of gravitational potential energy. Trivedi et al. (2007b) developed a model for trunk-like robots by using the Cosserat-rod approach, taking into account extension and shear (see Figure 12). Likewise, Boyer et al. (2006) used geometrically exact beam theory and the Newton-Euler technique to predict control torques of an eel-like robot as a function of expected internal deformations.

Complexity makes material model development difficult for soft EAP robots (Bar-Cohen 2000). Many researchers (e.g. Otake et al. 2002, Rajamani et al. 2005) have developed models to predict the performance of particular EAP actuators. Madden et al. (2007) compiled an online database of experimental methods and results (mechanical, electrical, chemical and other properties) for EAP-based actuators to facilitate actuator selection for design.

The inherent nonlinearity in underlying materials, geometry and actuators due to large strain and displacement makes modelling a challenging task. The soft structure makes the effect of distributed loading significant. Exclusion of these factors from modelling leads to unacceptable levels of inaccuracy, and their inclusion makes modelling computationally too expensive to be of practical use. Development of fast and accurate models for soft robots needs further study.

\subsection{Control}

To achieve point-to-point limb movements, the animal nervous system generates a sequence of motor commands that move the limb toward the target. In muscular hydrostats, this process is complicated because of the virtually infinite number of df (Sumbre et al. 2001). Reducing the number of $\mathrm{df}$ through coordination is a key problem in motor control of hyper-redundant limbs. Gutfreund et al. (1996) studied octopus arm movements to identify general principles of control. They concluded that the octopus actuates its arm for locomotion, reaching for objects or searching by a wave-like propagation of arm stiffening that travels from the base of the arm toward the tip. The region of stiffening tends to move within a plane in a slightly curved path connecting the centre of the animal's body with the target location. These authors proposed that this strategy reduces the immense redundancy of the octopus arm movements and hence simplifies motor control. Sumbre et al. (2005b) concluded that octopuses use strategies similar to vertebrates for transferring an object from one place to another The octopus temporarily configures its arm into a stiffened, articulated, quasi-jointed structure based on three dynamic joints. Rotational movements around these joints bring the object to the mouth. Certain kinematic characteristics in octopus arm motion such as relationship among rotation angles along the arm remain invariant at the joint level rather than at the end-effector level, suggesting intrinsic control co-ordination. This indicates that a kinematically constrained articulated limb may provide an optimal solution for precise point-to-point movements (Sumbre et al. 2005a).

Yekutieli et al. (2005) showed that a simple command producing a wave of muscle activation moving at a constant velocity is sufficient to replicate the natural reaching movements of octopus arms with similar kinematic features. Sumbre et al. (2001) showed experimentally that the basic motor program for voluntary movement of octopus arm is embedded within the neural circuitry of the arm itself. Such peripheral motor programs represent considerable simplification in the motor control of these appendages.

Wormlike robots employ sequential control input from tail to head, resulting in successive bending of the body to 


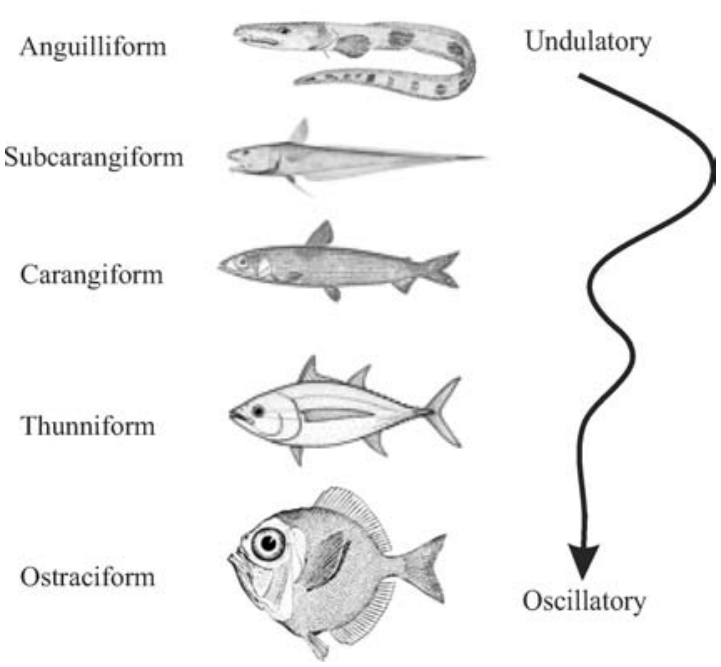

Figure 13. Body/caudal fin propulsion $(\mathrm{BCF})$ modes used for swimming robots.

create the travelling wave observed in natural undulatory motion. Cellular neural networks (CNNs) create central pattern generators (CPGs) that propagate the travelling wave for locomotion control (Arena et al. 2006). Each CNN cell is a non-linear oscillator that is coupled to the other oscillators and controls an actuator, acting as a motor neuron of the CPG. Swimming robots often mimic fish dynamics for locomotion, using, for example, body/caudal fin propulsion (see Figure 13). Tadpole (Jung et al. 2003) and eel (Jalbert et al.1995; Ijspeert and Kodjabachian 1999) robots use undulatory anguilliform locomotion. Anguilliform locomotion is found in some long, slender fish such as eels, in which the whole body is displaced laterally and there is little increase in the amplitude of the flexion wave as it passes along the body. The swimming robot of Guo et al. (2003) emulates carangiform locomotion, in which movement is restricted to the rear of the body and tail. Robo Tuna (2001) uses thunniform locomotion for high-speed and long-distance swimming, in which the lateral movement is in the tail and the region connecting the main body to the tail. Laurent and Piat (2001) have shown theoretically that robot fish should use undulatory motion rather than oscillatory motion (e.g. ostraciiform locomotion; Sfakiotakis et al. 1999) to obtain best performance. McIsaac and Ostrowski (2003) presented a dynamic model of anguilliform swimming for eel-like robots. Swimming gaits for forward and sideways swimming, turning and following circular paths are developed by using perturbation analysis.

The IPMC-based robot developed by Kim et al. (2003) is an example of a soft robot that locomotes on the ground by using ciliary motion. In each cycle, the front and the rear legs are alternately pushed downward and folded upward.

A motor scheme for the control of a single-joint robot arm actuated by McKibben artificial muscles is proposed by Eskiizmirliler et al. (2002). In this approach, classical control elements of the cybernetic circuit are replaced by artificial neural network modules having an architecture based on the connectivity of the cerebellar cortex and whose functioning is regulated by reinforcement learning. After learning, the model accurately pilots the movements of the robot arm, both in velocity and position.

Chitrakaran et al. (2007) and Hannan Walker (2005) proposed the use of an external camera for shape estimation and setpoint regulation of soft robotic arms. To simplify the inverse kinematics, Gravagne and Walker (2000a) proposed mapping infinite-dimensional arm configuration space to the finite-dimensional actuator space by using natural and wavelet decompositions. They used manipulability and force ellipsoids (Gravagne and Walker 2004) to analyse the directional dependence of motion and forceexerting capabilities of soft robotic manipulators. Gravenge and Walker (2002) showed that under the assumption that distributed damping exists on the backbone of a soft robotic manipulator, a PD plus feed-forward controller can exponentially regulate the manipulator configuration. Braganza et al. (2006) presented a combination of conventional controller with neural network-based learning for OctArmtype manipulators. Gravagne et al. (2001) formulated a vibration-damping setpoint controller. Otake et al. (2003) simplified the inverse dynamics of gel robots by selection of a central point on the robot and controlling the trajectory of that point.

Accurate control of soft robots requires model-based prediction of the set of possible configurations. Dynamic models that accurately describe large-scale deflections of soft robots and cover their entire workspace are currently too complicated to be used for control. Current control approaches, based on simpler models, are not guaranteed to be stable or effective for large deflections (Gravagene et al. 2001). Also, including distributed forces such as gravity, and structural stability of multiple section robots into control schemes is a challenging problem.

\subsection{Path planning}

Path planning for soft robots involves deforming soft appendages to conform to the environment or navigate through confined spaces. Most higher animals navigate by using cognitive maps based on current perceptions, memorised events and expected consequences (Tolman 1948). An enhanced navigation strategy can dramatically improve the locomotive capabilities of an organism. In soft biological appendages, path planning is complicated by the fact that multiple appendage shapes can achieve the same tip position and orientation. The processes by which animals choose simple paths are not well understood (Hooper 2006). It has been proposed (Sabes 2000; Wolpert and Ghahramani 2000; Flash and Sejnowski 2001) that those paths are chosen that balance motor command amplitude and endpoint 


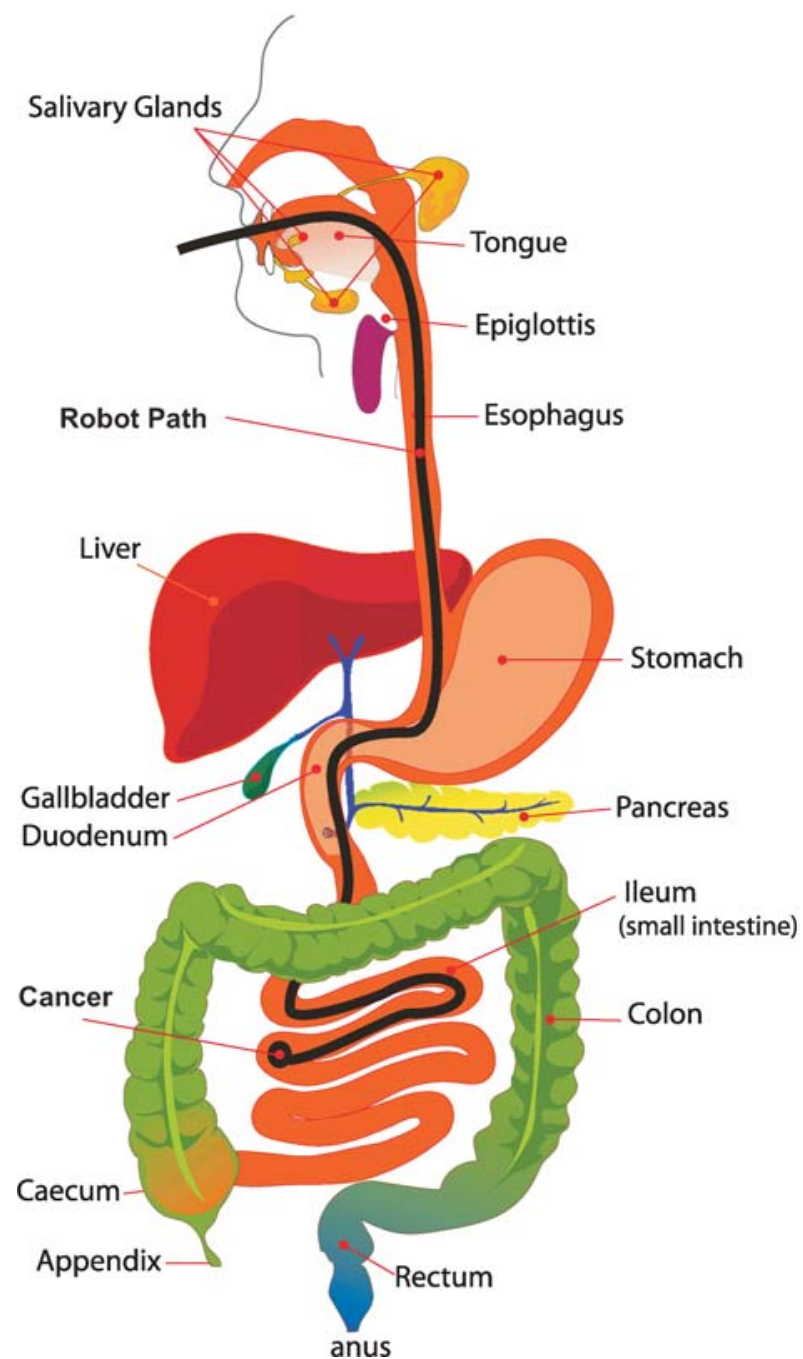

Figure 14. Path planning for reaching a cancer in the small intestine from the oral cavity.

tracking, leading to smooth and direct paths involving minimal motor commands and endpoint error.

Several researchers have attacked the problem of path planning for deformable objects such as routing a soft surgical tool into the small intestine as shown in Figure 14. Conru (1994) used a genetic algorithm to find near optimal routes for cables but did not include constraints imposed by the physical characteristics of the cable or the environment. Several researchers used a probabilistic roadmap (PRM) strategy for path planning of deformable objects (Halleman et al. 1998; Guibas et al. 1999; Lamiraux and Kavaraki 2001). In this approach, a large number of initial configurations are randomly generated and collision-free configurations with low energy are retained as roadmap nodes. Once a dense roadmap has been generated, the planner can answer queries by connecting initial and final configurations by searching a path. Holleman et al. (1998) and Lamiraux and Kavraki (2001) presented a probabilistic roadmap planner capable of finding paths in an obstacle field by using a low-degree Bezier surface patch and an approximate energy function that penalises deformation. Guibas et al. (1999) described an improved probabilistic algorithm for a surface patch by using the medial axis of the workspace to guide the random sampling. Prior to path planning, the medial axis of the workspace is computed and the flexible object is fitted at random points along the medial axis. The energy of all generated configurations is minimised and the planner connects them with low-energy quasi-static paths along the probabilistic roadmap.

Anshelevich et al. (2000) presented a path-planning algorithm for deformable volumes such as pipes and cables that uses a lumped-parameter model. This approach imposes constraints on the deformation to reduce complexity and does not work on general deformable objects. Bayazit et al. (2002) computed an approximate path and then refined the path by applying geometric-based free-form deformation to the robot. The approximate path can penetrate obstacles. The refined path is deformed to resolve any collisions. Gayle et al. (2005) presented a motion planning algorithm for simple closed robots that computes an approximate path between the initial and final configurations by using the probabilistic roadmap method. Constraint-based planning is applied to make appropriate path adjustments and corrections to compute a collision-free path. The algorithm takes into account geometric constraints such as nonpenetration and physical constraints such as volume preservation. Gayle et al. (2005), presented a fast algorithm for collision detection between a deformable robot and fixed obstacles that is used for path planning for a flexible robot in complex environments. The algorithm handles complex deformable models composed of tens of thousands of polygons.

Moll and Kavraki (2006) approached path planning as a constrained minimisation problem in which the planner is restricted to configurations that correspond to minimalenergy curves. The path planner computes paths from one minimal-energy curve to another such that all intermediate curves are also minimal-energy curves.

The path-planning approaches available for soft robots suffer from serious limitations, which make their practical use difficult at this time. Probabilistic methods tend to be computationally expensive because they require the generation of points in the configuration space, which grows exponentially with df. Available methods do not take into account the constraints imposed by the physical properties of the robots. Most planners work only in specific cases and do not work on general deformable objects.

\section{Future research}

The continued advancement of soft robotics depends on the development of novel soft sensors and actuators, soft robot designs with mobility and strength, models that enable design optimisation and control and fabrication techniques 
that build active soft structures and interconnections. Active materials currently available for use in soft robotic manipulators have shortcomings that make their commercial use impractical. New active materials are needed that provide the strain, stress and speed for this challenging application. Materials research alone, however, will not produce material in sufficient quantities to develop macro-scale actuators. Material science and engineering is required to produce bulk quantities of high quality active material and reliably fabricate high-performance actuators and sensors. Fabrication of soft robots also poses several challenges. Traditional rigid connectors (e.g. metallic fasteners) and electrodes cannot be used with soft structures, so new ways to connect actuators both mechanically and electrically must be developed.

With novel actuators available to the robot designer, new soft robots can, in principle, be designed that provide outstanding mobility, strength and reliability. The design process, however, is complicated owing to several competing and difficult-to-define design objectives. For example, there is often a trade-off between providing sufficient dexterity and maximising load capacity, both of which are design objectives for soft robotic manipulators (Dienno 2006, Trivedi et al. 2007a). There are many definitions of dexterity and load capacity, and the final design may depend significantly on these definitions. Although soft manipulators are capable of performing a wide variety of tasks because of their flexibility, designing a manipulator that performs optimally for an entire range of tasks is difficult.

Biological continuum manipulators mostly rely on hard/discrete elements in their structure and/or operation to reduce complexity in interaction with their environment, and similarly, a judicious mixture of continuous/soft and discrete/hard elements would significantly improve the performance of these robots in most applications (Cowan and Walker 2008).

To enable rapid virtual prototyping of soft robots, accurate physical models are needed. The design can be optimised prior to fabrication and accurately controlled on the basis of these models. Development of models that accurately simulate the operation of these robots on the basis of the actuation inputs is a challenging multiphysics problem that can involve simultaneous analysis of solid and fluid mechanics, kinematics, electromechanics, thermodynamics and chemical kinetics of the processes involved. A greater understanding of these phenomena would facilitate the development of accurate models and lead to better design and control.

Sensing and controlling the shape and motion of soft robots are problem that must be addressed rigorously. Soft robots theoretically have infinite df, but the number of sensors and actuators in any practical soft robot is finite. Therefore, many df of soft robots are not directly observable and/or controllable. Models that accurately describe the large-deflection dynamics of soft robots are generally too expensive computationally to be useful for real-time control. Appropriate model order reduction and efficient solution techniques may lead to fast yet accurate dynamic models that would be useful for control. Inverse dynamics models will be useful in the development of reliable feedforward control systems for soft robots.

An important issue in practice is user and operator interfaces for soft robots. User interfaces for soft robots are in their infancy. Soft robot structure and movements are quite different from those of humans, and human operators often become confused and disoriented. Although there has been some insightful early work in the area for soft manipulators (Csencsits et al. 2005), there is a strong need for more focused efforts in human factors, as well as hardware and software designs for operator feedback and input devices for soft robots.

New approaches in path planning that are computationally efficient for deformable bodies with infinite df are required to make real-time path-planning viable. This would require integration of the continuum mechanics of the robot into the path-planning algorithm to directly obtain feasible paths instead of generating a dense roadmap with a large number of random paths, and then searching a feasible one. Integrating motion planning with control and sensing is also an open problem.

Grasping objects by using whole arm manipulation requires the grasp to be stable so that the arm does not undergo sudden changes in shape and drop the manipulated object. Robust path-planning and control algorithms must ensure the stability of all intermediate configurations of the manipulator along the prescribed path. Extensive literature exists for grasp synthesis and stability analysis of rigid-link robots that contact objects only at finite points (Baker et al. 1985; Fearing 1986; Markenscoff et al. 1990; Bicchi 1993; Ismaeil and Eills 1994; Jen et al. 1996; Xiong et al. 2005). Stable grasp synthesis for highly compliant and continuously deformable soft manipulators, however, will require solutions to many untouched and challenging design, control and planning problems (Salisbury et al. 1988).

\section{References}

Alici G, Huynh NN. 2006. A robotic gripper based on conducting polymer actuators. Paper presented at: AMC'06. Istanbul, Turkey: IEEE, pp. 472-477.

Alici G, Huynh NN. 2007. Performance quantification of conducting polymer actuators for real applications: a microgripping system. IEEE/ASME Trans Mechatron. 12:73-84.

Anshelevich E, Owens S, Lamiraux F, Kavraki LE. 2000. Deformable volumes in path planning applications. Int Conf Robotics Automation. 3:2290-2295.

Arena P, Bonomo C, Fortuna L, Frasca M, Graziani S. 2006. Design and control of an ipmc wormlike robot. IEEE Tran Sys Man Cybernet—Part B: Cybernet. 36(5):1044-1052.

Baker BS, Fortune S, Grosse E. 1985. Stable prehension with a multi-fingered hand. In at: Proceedings of IEEE International 
Conference on Robotics Automation; St. Louis, USA: IEEE, p. 570-575.

Bar-Cohen Y. 2000. Electro-active polymers as artificial muscles - capabilities, potentials and challenges, Handbook on Biomimetics, Y. Osada, Ed. Peachtree City, GA: NTS Inc. 8:134.

Bayazit OB, Lien H, Amato N. 2002. Probabilistic roadmap motion planning for deformable objects. Int Conf Robotics Automation. Washington, DC, USA: IEEE, 2:2126-2133.

Bicchi A. 1993. Force distribution in multiple whole limb manipulation. Paper presented at: Proceedings of the IEEE International Conference on Robotics and Automation. Atlanta, Georgia, USA: IEEE, 228-233.

Boyer F, Porez M, Khalil W. 2006. Macro-continuous computed torque algorithm for a three-dimensional eel-like robot. IEEE Trans Robotics. 22:763-775.

Braganza D, Dawson DM, Walker ID, Nath, N. 2006. Neural network grasping controller for continuum robots. In: 45th IEEE Conference on Decision and Control. San Diego: IEEE, 6445-6449.

Buckingham R, Graham A. 2003. Reaching the unreachablesnake-arm robots. Paper Presented at: International Symposium of Robotics. Available from: OCR obotics Ltd. Chicago, USA: International Federation of Robotics, 3-Sep08, http://www.ocrobotics.com

Chapman G. 1958. The hydrostatic skeleton in the invertebrates. Biol. Revi. Cambridge Philos Soc. 33:338-371.

Chapman G. 1975. Versatility of hydraulic systems. J Exp Zool. 194:249-270.

Chen I-M, Li H-S, Cathala A. 1999. Design and simulation of amoebot-a metamorphic underwater vehicle, Detroit, Michigan, USA: IEEE, IEEE Inter Conf Robotics Automation. 1:90-95.

Chiel HJ, Crago PE, Mansour JM, Hathi K. 1992. Biomechanics of a muscular hydrostat: a model of lapping by a reptilian tongue, Biol Cybernet. 67:403-415.

Chirikjian GS. 1993. Continuum approach to hyper-redundant manipulator dynamics. Proceedings of International Conference on Intelligent Robotic Systems. Tokyo, Japan: IEEE, pp.1059-1066.

Chirikjian GS. 1995. Hyper-redundant manipulator dynamics: a continuum approximation, Adv. Robotics. 9(3):217-243.

Chirikjian GS. 1997. Inverse kinematics of binary manipulators using a continuum model, J Intell Robotic Syst: Theory Appli. 19:5-22.

Chitrakaran VK, Behal A, Dawson DM, Walker ID. 2007. Setpoint regulation of continuum robots using a fixed camera. Robotica. 25:581-586.

Cieslak R, Morecki A. 1999. Elephant trunk type elastic manipulator-a tool for bulk and liquid materials transportation. Robotica. 17:11-16.

Clark RB. 1964. Dynamics in Metazoan Evolution. The origin of the coelom and segments. Oxford: Clarendon Press.

Clark RB, Cowey JB. 1958. Factors controlling the change of shape of certain nemertean and turbellarian worms. J Exp Zool. 35:731-748.

Conru A. 1994. A genetic approach to the cable harness routing problem. Proceedings of the IEEE World Congress on Computation Intelligence. Orlando, FL: IEEE, 1:200205.

Cortés MT, Moreno JC. 2003. Artificial muscles based on conducting polymers. e-Polymers. 41:1-42.

Cowan LS, Walker ID. 2008. 'soft' continuum robots: the interaction of continuous and discrete elements. In: Euro. Conference of Artificial Life XI. Boston: MIT Press, p.126-133.
Crespi A, Ijspeert AJ. 2006. Amphibot II: an amphibious snake robot that crawls and swims using a central pattern generator. Paper presented at: 9th International Conference on Climbing and Walking Robots (CLAWAR 2006). Brussels, Belgium: RMA Polytechnics, p. 19-27.

Csencsits M, Jones B, McMahan W, Walker I. 2005. User interfaces for continuum robot arms. In. Proc. IEEE/RSJ Intl. Conf. on Intelligent Robots and Systems; Edmonton, Canada: IEEE, p. 3011-3018.

Daerden F, Lefeber D. 2002. Pneumatic artificial muscles: actuators for robotics and automation. Eur J Mech Environ Eng. 47(1):11-21.

Daugela A, Jeronymo HFCE, Misaki A. 1995. Piezo ceramic based locomotive drive. Paper presented at: Sixth IEEE International Symposium on Micro Machine and Human Science. Nagoya, Japan: IEEE, p. 187-192.

Deng X. Avadhanula S. 2005. Biomimetic micro underwater vehicle with oscillating fin propulsion: system design and force measurement. In: Proceedings of the 2005 IEEE International Conference on Robotics and Automation Barcelona, Spain: IEEE, April 2005. p. 3312-3317.

Dienno DV. 2006. Design and analysis of a soft robotic manipulator with base rotation [master's thesis]. University Park: The Pennsylvania State University.

Eckerle J, Stanford S, Marlow J, Schmidt R, Oh S, Low T, Shastri SV. 2001. Biologically inspired hexapedal robot using fieldeffect electroactive elastomer artificial muscles. McGowan A-MR, editor. In: Smart Structures and Materials 2001: Industrial and Commercial Applications of Smart Structures Technologies. A.-M. R. McGowan, editor. Proce. SPIE, 4332:269280.

Eskiizmirliler S, Forestier N, Tondu B, Darlot C. 2002. A model of the cerebellar pathways applied to the control of a singlejoint robot arm actuated by McKibben artificial muscles. Biol Cybernet. 86(5):379-394.

Fearing RS. 1986. Simplified grasping and manipulation with dexetrous robot hands. IEEE J Robot Automation. 2:188-195.

Festo. 2007. Airacuda - the remote controlled, pneumatically driven fish. [cited 11-Nov-08] Available from: http://www. festo.com/INetDomino/coorp_sites/en/f1b00561e66a68b7c $12571 \mathrm{~b} 9002 \mathrm{~b} 67 \mathrm{c} 3 . \mathrm{htm}$

Flash T, Sejnowski T. 2001. Computational approaches to motor control. Curr Opin Neurobiol. 11:655-662.

Gayle Lin M, Manocha D. 2005. Constraint-based motion planning of deformable robots, Proceedings of Robotics: Systems and Science, 2005. IEEE Conf Robot Automation. pp. 10461053.

Gayle R, Segars P, Lin M, Manocha D. 2005. Path planning for deformable robots in complex environments. Cambridge, Massachusetts: RSS.

Georgiades C, German A, Hogue A, Lid H, Prahacs C, Ripsman A, Simit R, Tomsti L-A, Zhangt P, Buehler M. 2004. Aqua: an aquatic walking robot. In: Proceedings of the 2005 IEEE International Conference on Intelligent Robots and Systems; Sendai, Japan: IEEE, p. 3525-3531.

Gerard. 2003. A 3d biomechanical tongue model to simulate speech movements. Arch Physiol Biochem. 111:1381-3455.

Gravagne IA, Rahn CD, Walker ID. 2001. Good vibrations: a vibration damping setpoint controller for continuum robots. In: Proceedings of the 2001 IEEE International Conference on Robotics 8 Automation; Seoul, Korea: IEEE, 21-26, May 2001. p. 3877-3884.

Gravagne IA, Rahn CD, Walker ID. 2003. Large deflection dynamics and control for planar continuum robots. In: IEEE/ASME Trans Mechatron. 8:299-307. 
Gravagne IA, Walker ID. 2000a. Kinematic transformations for remotely actuated planar continuum robots. IEEE International Conference on Intelligent Robots and Systems; San Francisco, CA, USA: IEEE, p. 19-26.

Gravagne IA, Walker ID. 2002. Uniform regulation of a multisection continuum manipulator. In: IEEE International Conference on Intelligent Robots and Systems; San Francisco, CA, USA: IEEE, p. 1519-1524.

Gravagne IA, Walker ID. 2004. Manipulability and force ellipsoids for continuum robot manipulators. In: IEEE International Conference on Intelligent Robots and Systems. Sendai, Japan: IEEE, p. 304-311.

Gravagne I, Walker ID. 2000b. On the kinematics of remotelyactuated continuum robots. Proc-IEEE Int Conf Robot Automat. San Francisco, USA: IEEE, 3:2544-2550.

Guibas L, Holleman C, Kavraki L. 1999. A probabilistic roadmap planner for flexible objects with a workspace medial-axisbased sampling approach. International Conference on Intelligent Robots and Systems. Kyongju, Korea: IEEE, p. 254 260.

Guo S, Fukuda T, and Asaka K. 2003. A new type of fish-like underwater microrobot. IEEE/ASME Trans Mechatron. 8:136141.

Gutfreund Y, Flash T, Yarom Y, Fiorito G, Segev I, Hochner B. 1996. Organization of octopus arm movements: a model system for studying the control of flexible arms. J Neurosci. 16:7297-7307.

Gutmann WF. 1981. Relationships between invertebrate phyla based on functional-mechanical analysis of the hydrostatic skeleton. Am Zool. 21:63-81.

Hannan M, Walker ID. 2000a. Analysis and initial experiments for a novel elephantòs trunk robot. Paper presented at: Proc. Int. Conf. on Intelligent Robots and System (IROS). San Francisco, USA: IEEE, p. 330-337.

Hannan, M. Walker ID. 2000b. Novel kinematics for continuum robots. In: 7th Int. Symp. Advances in Robot Kinematics. p. $227-238$

Hannan M, Walker ID. 2001. Analysis and experiments with an elephant's trunk robot. Adv Robot. 15:847-858.

Hannan M, Walker ID. 2003. Kinematics and the implementation of an elephant's trunk manipulator and other continuum style robots. J Robot Syst. 20:45-63.

Hannan M, Walker ID. 2005. Real-time shape estimation for continuum robots using vision. Robotica 23:645-651.

Harris JE, Crofton HD. 1957. Structure and function in the nematodes: internal pressure and cuticular structure in ascaris. J Exp Biol 34:116-130.

Herrel A, Meyers JJ, Aerts P, Nishikawa KC. 2001. Functional implications of supercontracting muscle in the chameleon tongue retractors. J Exp Biol 204:3621-3627.

Hetherington A. 2001. Guard cells. Curr Biol. 11(15):R588.

Hirose S. 1993. Biologically inspired robots. Oxford University Press. p. 147-155.

Hodick D, Sievers A. 1989. On the mechanism of trap closure of venus flytrap (Dionaea muscipula Ellis). Planta 179: $32-42$.

Holleman C, Kavraki LE, Warren J. 1998. Planning paths for a flexible surface patch, IEEE Int Conf Robotics Automat. Leuvan, Belgium: IEEE, 1:21-26.

Hooper SL. 2006. Motor control: the importance of stiffness. Curr. Biol. 16(8):R283-R285.

Hunter IW, Lafontaine S. 1992. Comparison of muscle with artificial actuators. In: IEEE Solid-State Sensor and Actuator Workshop. Hilton Head Island, SC: IEEE, p. 178185.
Ijspeert AJ, Kodjabachian J. 1999. Evolution and development of a central pattern generator for the swimming of a lamprey. Artif Life 5:247.

Immega G, Antonelli K. 1995. The ksi tentacle manipulator. In: IEEE Int Conf Robotics Automation. Nagoya, Aichi, Japan: IEEE, p. 3149-3154.

Ismaeil OM, Ellis RE. 1994. Grasping using the whole finger. Int Conf Robot Automat. San Diego, CA: IEEE, 4:3111-3116.

Jalbert J, Kashin S, Ayers J. 1995. A biologically based undulatory lamprey-like auv. In: Proc. of the Autonomous Vehicles in Mine Countermeasures Symposium. Montery, CA: US Military, p. 39-52.

Jen F, Shoham M, Longman RW. 1996. Liapunov stability of forcecontrolled grasps with a multi-fingered hand. Int J Robot Res. 15:137-154.

Jones BA, Walker ID. 2006. Kinematics for multisection continuum robots. IEEE Trans Robot. 22:43-55.

Jung J, Tak Y, Kim B, Park J-O, Lee S-K, Pak J. 2003. Tadpole robot (TadRob) using ionic polymer metal composite (IPMC) actuator. In: Y. Bar-Cohen, editor, Smart Structures and Materials 2003: Electroactive Polymer Actuators and Devices (EAPAD). Proce SPIE 5051: Bellingham, WA: SPIE Press, p. 272-280.

Jung K, Koo1 JC, do Nam J, Lee YK, Choi HR. 2007. Artificial annelid robot driven by soft actuators. Bioinspir Biomimet 2:S42-S49.

Kanzawa N, Hoshino Y, Chiba M, Hoshino D, Kobayashi H, Kamasawa N, Kishi Y, Osumi M, Sameshima M, Tsuchiya T. 2006. Change in the actin cytoskeleton during seismonastic movement of Mimosa pudica. Plant Cell Physiol. 47:531-539.

Kerrebrock PA, Anderson JM, Parry JR. 2001. Application requirements of artificial muscles for swimming robots. In BarCohen, Y., editor, Proc. SPIE Vol. 4329, p. 364-374, Smart Structures and Materials 2001: Electroactive Polymer Actuators and Devices. Paper In: Proceedings the Presented at the 4329: Society of Photo-Optical Instrumentation Engineers (SPIE) Conference. Bellingham, WA: SPIE Press, p. 364-374.

Kier WM. 1987. The functional morphology of the tentacle musculature of Nautilus pompilius. Plenum Publishing Corporation.

Kier WM. 1989. The fin musculature of cuttlefish and squid (mollusca, cephalopoda): morphology and mechanics. J. Zool. (London) 217:23-38.

Kier WM, Curtin NA. 2002. Fast muscle in squid (Loligo pealei): contractile properties of a specialized muscle fibre type. J Exp Biol 205:1907-1916.

Kier WM, Smith, AM. 1990. The morphology and mechanics of octopus suckers. Biol. Bull. 178:126-136.

Kier WM, Smith KK. 1985. Tongues, tentacles, and trunks: the biomechanics of movement in muscular-hydrostats. Zool $\mathrm{J}$ Linnean Soc. 83:307-324.

Kier WM, Stella MP. 2007. The arrangement and function of octopus arm musculature and connective tissue. J Morphol 268:831-843.

Kier WM, Thompson JT. 2003. Muscle arrangement and function, and specialization in recent coleoids. Berliner Palobiol Abhandlungen. 3:141-162.

Kim B, Ryu J, Jeong Y, Tak Y, Kim B, Park JO. 2003. A ciliary based 8-legged walking micro robot using cast ipmc actuators. Proceedings of the 2003 IEEE International Conference on Robotics and Automation, September 2003, Taipei, Taiwan: IEEE.

Klute GK, Czerniecki JM, Hannaford B, 1999. McKibben artificial muscles: pneumatic actuators with biomechanical intelligence. In: Proceedings of IEEE/ASME International 
Conference on Advanced Intelligent Mechatronics. Atlanta, Georgin, USA: IEEE, p. 221-226.

Kornbluh R, Pelrine R, Eckerle J. Joseph J. 1998. Electrostrictive polymer artificial muscle actuators. In: Proceedings of the 1998 IEEE International Conference on Robotics and Automation. Leuven, Belgium: IEEE, p. 2147-2154.

Kukolj M. 1988. Axially contractible actuator, US Patent No. 4733603 .

Lachat D, Crespi A, Ijspeert AJ. 2006. Boxybot: a swimming and crawling fish robot controlled by a central pattern generator. In: Proceedings of The first IEEE/RAS-EMBS International Conference on Biomedical Robotics and Biomechatronics (BioRob 2006). Pisa, Italy: IEEE, p. 643-648.

Lamiraux F, Kavraki L. 2001. Path planning for elastic objects under manipulation constraints, Int J Robot Res. 20(2):188208.

Laurent G, Piat E. 2001. Efficiency of swimming microrobots using ionic polymer metal composite actuators, In: Proceedings of the 2001 IEEE International Conference on Robotics and Automation; Seoul, Korea: IEEE, Vol. 4; p. 3914-3919.

Liang Y, McMeeking R, Evans A. 2006. A finite element simulation scheme for biological muscular hydrostats. J Theor Biol. 242:142-150.

Liu J, Hu H, Gu D. 2006. A layered control architecture for autonomous robotic fish. In: Proc. IEEE/RSJ Intl. Conf. on Intelligent Robots and Systems. 9-15 October 2006; Beijing, China: IEEE, p. 312-317.

Madden JDW, Vandesteeg NA, Anquetil PA, Madden PGA, Takshi A, Pytel RZ, Lafontaine SR, Wieringa PA, Hunter IW. 2004. Artificial muscle technology: physical principles and naval prospects. IEEE J. Oceanic Eng. 29:706-728.

Madden J, Genereux N, Shkuratoff K, Star AVD, Poon D, Irwin D, Cheng P, Hsu G, Filipozzi, L. 2007. Electroactive polymer actuator online database. In. Proceedings of SPIE-The International Society for Optical Engineering. Bellingham, WA: SPIE Press p. 65240P.

Mangan E, Kingsley D, Quinn R, Chiel H. 2002. Development of a peristaltic endoscope. IEEE Int Conf on Robot Automat. Washington DC, USA: IEEE, 1:347-352.

Markenscoff X, Ni L, Papadimitriou CH, 1990. The geometry of grasping. Int J Robot Res. 9:61-74.

McCurley RS, Kier WM. 1995. The functional morphology of starfish tube feet: the role of a crossed-fiber helical array in movement. Biol Bull. 188:197-209.

McIsaac K, Ostrowski J. 2003. Motion planning for anguilliform locomotion. Robot. Automat. IEEE Trans. 19(4):637652.

Meijer K, Bar-Cohen Y, Full RJ. 2003. Biological inspiration for musclelike actuators of robots and biologically inspired intelligent robots. Bellingham, WA: SPIE Press, pp. 2546.

Meijer K, Rosenthal M, Full RJ. 2001. Muscle-like actuators? A comparison between three electroactive polymers. Proc SPIE-Inter Soc Optical Eng. 4329. Bellingham WA: SPIE Press, 7-15.

Menciassi A, Dario P. 2003. Bio-inspired solutions for locomotion in the gastrointestinal tract: background and perspectives. Philos Trans R Soc. 361(1811):2287-2298.

Meyers JJ, O’Reilly JC, Monroy JA, Nishikawa KC. 1999. Mechanism of tongue protraction in microhylid frogs. J Exp Biol. 207:21-31.

Micromuscle. 2007. Available from: www.micromuscle.com 17 October 2008.

Miller G. 2007. Snake robots by Dr. Gavin Miller. Available from: www.snakerobots.com 11 November 2008.
Mochiyama H, Kobayashi H. 1998. Shape correspondence between a spatial curve and a manipulator with hyper degrees of freedom. In: Proc. IEEE/RSJ Int. Conf. Intelligent Robots and Systems (IROS), October 1998; Victoria, BC, Canada: IEEE, pp. 161-166.

Mochiyama H, Kobayashi H. 1999. The shape jacobian of a manipulator with hyper degrees of freedom flexible manipulator. Proc IEEE Int Conf Robot Automat. Detroit, Michigan, USA: IEEE, Vol. 2, p. 2837-2842.

Mochiyama H, Suzuki T. 2003. Kinematics and dynamics of a cable-like hyper-flexible manipulator. Proc IEEE Int Conf. Robot Automat. Taipei, Taiwan: IEEE, 3:3672-3677.

Mojarrad M, Shahinpoor M. 1997. Biomimetic robotic propulsion using polymeric artificial muscles. Proc. IEEE Int Conf Robot Automat. Albuquerque, New Mexico, USA: IEEE, Vol. 1, p. 2152-2157.

Moll M, Kavraki LE. 2006. Path planning for deformable linear objects. IEEE Trans Robot. 22(4):625-636.

Morin AH. 1953. Elastic diaphragm. US Patent No. 2642091.

Naciri J, Srinivasan A, Ratna BR. 2004. Liquid crystal elastomer for artificial muscle applications. In Bar-Cohen Y, editor. Smart Structures and Materials 2004: Electroactive Polymer Actuators and Devices (EAPAD). Proc SPIE. Bellingham WA: SPIE Press, 5385:548-554.

Nakabo Y, Mukai T, Asaka K. 2007. Electroactive polymers for robotic applications. London: Springer, Chapter 7, Biomimetic soft robots using IPMC.

Napadow VJ, Kamm RD, Gilbert RJ. 2002. A biomechanical model of sagittal tongue bending. J Biomech Eng. 124:547556.

Nie L, Li D, Guo S. 2006. Design and analysis of the dynamics of icpf actuated tortoise-like lexible micro-robot. Proc. SPIE Int Soc Optical Eng. Bellingham WA: SPIE Press, 6357:6357571-6357576.

Nishikawa KC, Kier WM, Smith KK. 1999. Morphology and mechanics of tongue movement in the African pig-nosed frog Hemisus marmoratum: a muscular hydrostatic model, J Exp Biol. 202:771-780.

O'Halloran A, O'Malley F. 2001. Materials and technologies for artificial muscle: a review for the mechatronic muscle project. Department of Electronic Engineering and National University of Ireland Galway chapter vii.

Ostrowski J, Burdick J. 1998. The geometric mechanics of undulatory robotic locomotion. Int J Robot Res. 17(7):683-701.

Otake M, Kagami Y, Inaba M, Inoue H. 2002. Motion design of a starfish-shaped gel robot made of electro-active polymer gel. Robot Auton Syst. 40:185-191.

Otake M, Kagami Y, Kuniyoshi Y, Inaba M, Inoue H. 2003. Inverse dynamics of gel robots made of electro-active polymer gel. In: IEEE International Conference on Robotics and Automation; Taipei, Taiwan. Pisa, Italy: IEEE, p. 2299-2304.

Pak NN, Scapellato S, Spina, GL, Pernorio G, Menciassi A, Dario P. 2006. Biomimetic design of a polychaete robot using IPMC actuator. In: Procedings of the First International Conference on Biomedical Robotics and Biomechatronics. Pisa, Italy: IEEE, Vol. 1, p. 666-671.

Paynter HM. 1988a. High pressure fluid-driven tension actuators and methods for constructing them. US Patent No. 4751869.

Paynter HM. 1988b. Hyperboloid of revolution fluid-driven tension actuators and methods of making. US Patent No. 4721030; p. 1 .

Pei Q, Pelrine R, Rosenthal M, Stanford S, Prahlad H, Kornblu, R. 2004. Recent progress on electroelastomer artificial muscles and their application for biomimetic robots. In: Bar-Cohen, editor. Smart Structures and Materials 2004: Electroactive 
Polymer Actuators and Devices (EAPAD). Proc SPIE. Bellingham WA: SPIE Press, 5385:41-50.

Pelrine R, Kornbluh R, Pei Q, Stanford S, Oh S, Eckerle J, Full R, Rosenthal M, Meijer K. 2002. Dielectric elastomer artificial muscle actuators: toward biomimetic motion. In: Bar-Cohen Y, editor. Smart Structures and Materials 2002: Electroactive Polymer Actuators and Devices (EAPAD). Proc SPIE. Bellingham WA: SPIE Press, Vol. 4695, p. 126-137.

Pritts MB. 2005. Design and static analysis of a soft robotic manipulator [master's thesis]. University Park: The Pennsylvania State University.

Pritts MB, Rahn CD. 2004. Design of an artificial muscle continuum robot. In: Proceedings-IEEE International Conference on Robotics and Automation. New Orleans, LA, USA: IEEE, p. $4742-4746$.

Rajamani A, Grissom M, Rahn C, Ma Y, Zhang Q. 2005. Wound roll dielectric elastomer actuators: Fabrication and analysis and experiments. In: IEEE/RSJ Int Conf Intell Robots Syst. Edmonton, Canada: IEEE, Vol. 2, p. 1520-1525.

Robinson G, Davies JBC. 1999. Continuum robots - a state of the art. IEEE International Conference on Robotics and Automation; May 1999. Detroit, Michigan, USA: IEEE, Vol. 4, p. 2849-2854.

RoboTuna. 2001. MIT Ocean Engineering, RoboTuna. Available from: http://web.mit.edu/towtank/www/Tuna/tuna.html

Sabes P. 2000. The planning and control of reaching movements. Curr Opin Neurobiol 10:740-746.

Salisbury K, Townsend W, Ebrman B, DiPietro D. 1988. Preliminary design of a whole-arm manipulation system (WAMS). Proc IEEE Int Conf. Philadelphia, PA, USA: IEEE, 254-260 vol.1.

Samatham R, Kim K, Dogruer D, Choi H, Konyo M, Madden J, Nakabo Y, Nam J, Su J, Tadokoro S, 2007. Electroactive polymers for robotic applications. London: Springer. Chapter 1, Active polymers: an overview.

Sears P, Dupont P. 2006. A steerable needle technology using curved concentric tubes. In 2006 IEEE/RSJ International Conference on Intelligent Robots and Systems. Beijing, China: IEEE, p. 2850-2856.

Sfakiotakis M, Lane DM, Davies JBC. 1999. Review of fish swimming modes for aquatic locomotion. IEEE J Oceanic Eng. 24(2):237-252.

Shahinpoor M, Bar-Cohen Y, Simpson JO, Smith J. 1998. Ionic polymer-metal composites (IPMCS) as biomimetic sensors and actuators and artificial muscles - a review. Smart Mater Struct. 7:R15-R30.

Shahinpoor M, Kim KJ. 2001. Ionic polymer-metal composites: I. Fundamentals. Smart Mater Struct. 10: 819-833.

Shiga TT, Hirose Y, Okada A, Kurauchi T. 1989. Bending of ionic polymer gel caused by swelling under sinusoidally varying electric fields. Jpn J Polyme Sci Technol. 46: 709.

Simaan N, Taylor R, Flint P. 2004. A dexterous system for laryngeal surgery. In Proceedings. ICRA'04. 2004 IEEE International Conference on Robotics and Automation. Barcelona, Spain: IEEE, Vol. 1, pp. 351-357.

Skierczynski BA, Wilson RJA, Kristan WB, Skalak R. 1996. A model of the hydrostatic skeleton of the leech. J Theor Biol. 181:329-342.

Sugiyama Y, Hirai S. 2006. Crawling and jumping by a deformable robot. I. J Robotic Res. 25(5-6):603-620.

Sumbre G, Fiorito G, Flash T, Hochner B. 2005a. Octopuses use a human-like strategy to control precise point-to-point arm movements. Curr Biol. 16:767-772.

Sumbre G, Fiorito G, Flash T, Hochner B. 2005b. Precise motor control of flexible arms. Nature 433:595-596.
Sumbre G, Gutfreund Y, Fiorito G, Flash T, Hochner B. 2001. Control of octopus arm extension by a peripheral motor program. Science 293:1845-1848.

Suzumori K, Endo S, Kanda T, Kato N, Suzuki H. 2007. A bending pneumatic rubber actuator realizing soft-bodied manta swimming robot. IEEE International Conference on Robotics and Automation. Rome, Italy: IEEE, Vol. 1, pp. 4975-4980.

Suzumori K, Iikura S, Tanaka H. 1991. Development of flexible microactuator and its application to robotic mechanisms. IEEE International Conference on Robotics and Automation. Sacramento, CA: IEEE, Vol. 1, pp. 1622-1627.

Tabata O, Hirasawa H, Aoki S. 2001. Ciliary motion on actuator using self-oscillating gel. In: Proceedings of the IEEE Micro Electro Mechanical Systems (MEMS). Interlaken, Switzerland: IEEE, Vol. 1, p. 405-408.

Tadokoro S, Yamagami S, Ozawa M, Kimura T, Takamori T, Oguro K. 1999. Multi-dof device for soft micromanipulation consisting of soft gelactuator elements. In: 1999 IEEE International Conference on Robotics and Automation, Detroit, Michigan, USA: IEEE, Vol. 3, p. 2177-2182.

Takagi S. 2002. Actin-based photo-orientation movement of chloroplasts in plant cells. J Exp Biol. 206:1963-1969.

Tatlicioglu E, Walker I, Dawson D. 2007. New dynamic models for planar extensible continuum robot manipulators. In: Proceedings of the 2007 IEEE International Conference on Intelligent Robots and Systems; San Diego, CA: IEEE, p. 1485-1490.

Taylor JRA, Kier WM. 2003. Switching skeletons: hydrostatic support in molting crabs. Science 301:209-210.

Taylor JRA, Kier WM. 2006. A pneumo-hydrostatic skeleton in land crabs. Nature 440:1005.

Tolman EC. 1948. Cognitive maps in rats and man. Psychol Rev. 55:189-208.

Trimmer B, Takesian A, Sweet B. 2006. Caterpillar locomotion: a new model for soft-bodied climbing and burrowing robots. Paper presented at: 7th International Symposium on Technology and the Mine Problem, Monterey, CA: Mine Warfare Association.

Trimmer B, Takesian A, Sweet B, Rogers C, Hake D, Rogers D. 2006. Caterpillar locomotion: a new model for soft-bodied climbing and burrowing robots. In 7th International Symposium on Technology and the Mine Problem. Monterey, CA: Mine Warfare Association, Vol. 1, p. 1-10.

Trivedi D, Dienno D, Rahn CD. 2007. Optimal, model-based design of soft robotic manipulators. Paper presented at: Proceedings of the 2007 ASME International Design Engineering Technical Conference, Las Vegas, NV: ASME.

Trivedi D, Lotfi A, Rahn CD. 2007. Geometrically exact dynamic models for soft robotic manipulators. Paper presented at: Proceedings of the 2007 IEEE International Conference on Intelligent Robots and Systems, San Diego, CA: IEEE.

Tsukagoshi H, Kitagawa A, Segawa M. 2001. Active hose: an artificial elephant's nose with maneuverability for rescue operation. In: IEEE International Conference on Robotics and Automation, Seoul, South Korea: IEEE, p. 2454-2459.

van Leeuwen J, Kier W. 1997. Functional design of tentacles in squid: linking sarcomere ultrastructure to gross morphological dynamics. Philoso Trans R Soc Lond B: Biol Sci. 352:551571.

Wainwright SA. 1982. Structural systems: hydrostats and frameworks. In companion to animal physiology. New York: Cambridge University Press.

Wainwright SA. 1988. Axis and circumference. Cambridge, (MA): Harvard University Press.

Wainwright SA, Vosburgh F, Hebrank JH. 1978. Shark skin: function in locomotion. Science 202:747-749. 
Webster RJ, Okamura AM, Cowan NJ. 2006. Toward active cannulas: miniature snake-like surgical robots. In: 2006 IEEE/RSJ International Conference on Intelligent Robots and Systems. Beijing, China: IEEE, p. 2857-2863.

Wei W, Xu K, Simaan N. 2006. A compact two-armed slave manipulator for minimally invasive surgery of the throat. In: BioRob 2006. The First IEEE/RAS-EMBS International Conference on Biomedical Robotics and Biomechatronics, Pisa, Italy: IEEE, 2006. p. 769-774.

Wilson JF, Li D, Chen Z, George RT. 1993. Flexible robot manipulators and grippers: relatives of elephant trunks and squid tentacles. In: Robots and biological systems: toward a new bionics? NJ, USA: Springer-Verlag New York, Inc., p. 474479.

Wilson JF, Mahajan U, Wainwirght SA, Croner LJ. 1991. A continuum model of elephant trunks. J Biomech Eng. 113: 79-84.

Wingert A, Lichter M, Dubowsky S, Hafez M. 2002. Hyperredundant robot manipulators actuated by optimized binary dielectric polymers. Smart Structures and Materials 2002:
Electroactive Polymer Actuators and Devices (EAPAD). Proceedings of SPIE, Vol. 4695, pp. 415-423.

Wolpert D, Ghahramani Z. 2000. Computational principles of movement neuroscience. Nat Neurosci. Suppl 3:1212-1217.

Wright C, Johnson A, Peck A, McCord Z, Naaktgeboren A, Gianfortoni, P, Gonzalez-Rivero M, Hatton R, Choset H. 2007. Design of a modular snake robot. In: IROS 2007. IEEE/RSJ International Conference on Intelligent Robots and Systems, San Diego, CA: IEEE, 2007. p. 2609-2614.

Xiong C-H, Wang MY, Tang Y, Xiong Y-L. 2005. Grasping matrix-compliant grasping with passive forces. J Robot Sys 22:271-285

Yarlott JM. 1972. Fluid actuator. US Patent No. 3645173.

Yekutieli Y, Sagiv-Zohar R, Aharonov R, Engel Y, Hochner B, Flash T. 2005. Dynamic model of the octopus arm. I. Biomechanics of the octopus reaching movement. J Neurophysiol 94:1443-1458.

Zhang X, Lowe C, Wissler CM, Jahne B, Kovacs G. 2005. Dielectric elastomers in actuator technology. Adv Eng Mater. 7(5):361-367. 

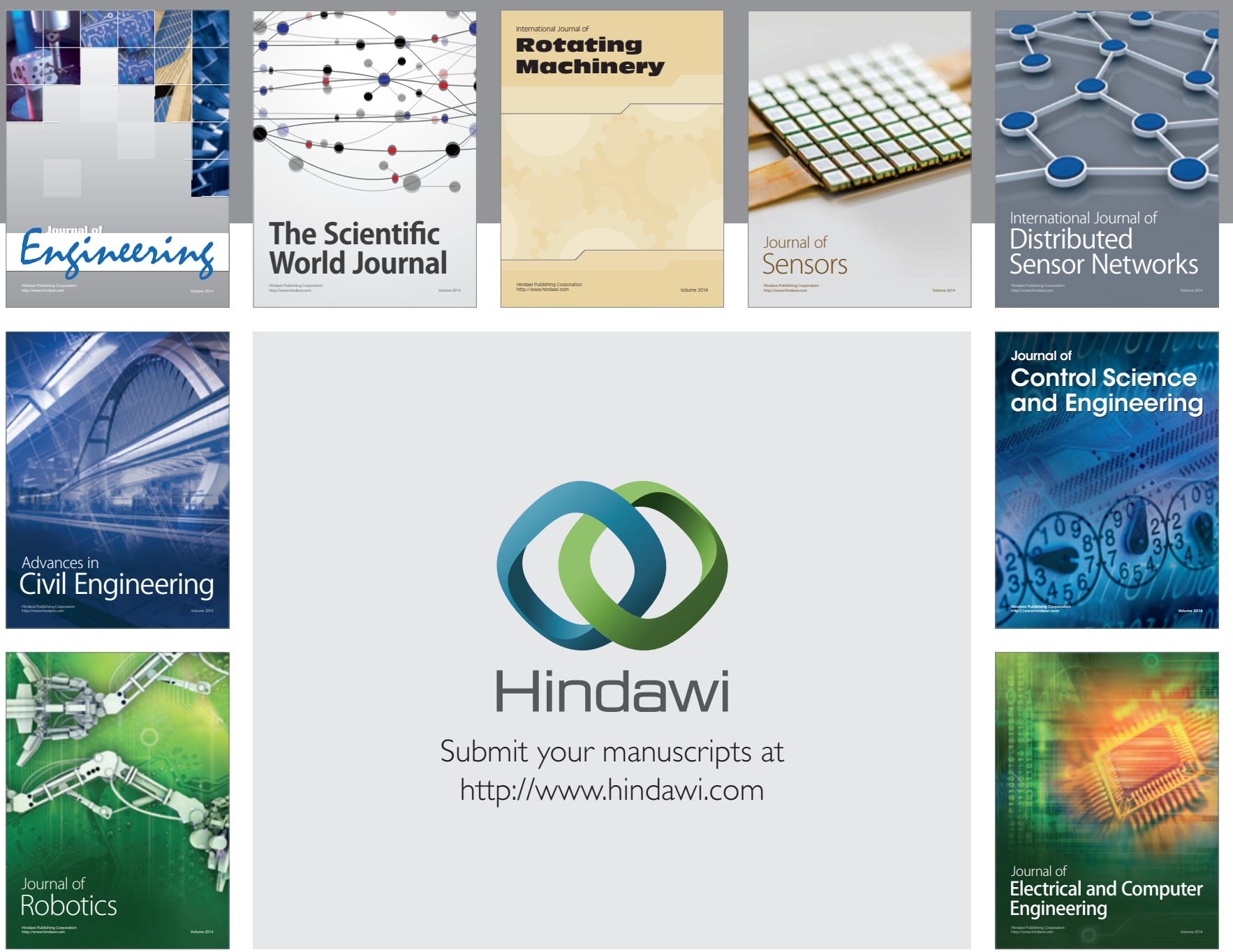

Submit your manuscripts at

http://www.hindawi.com
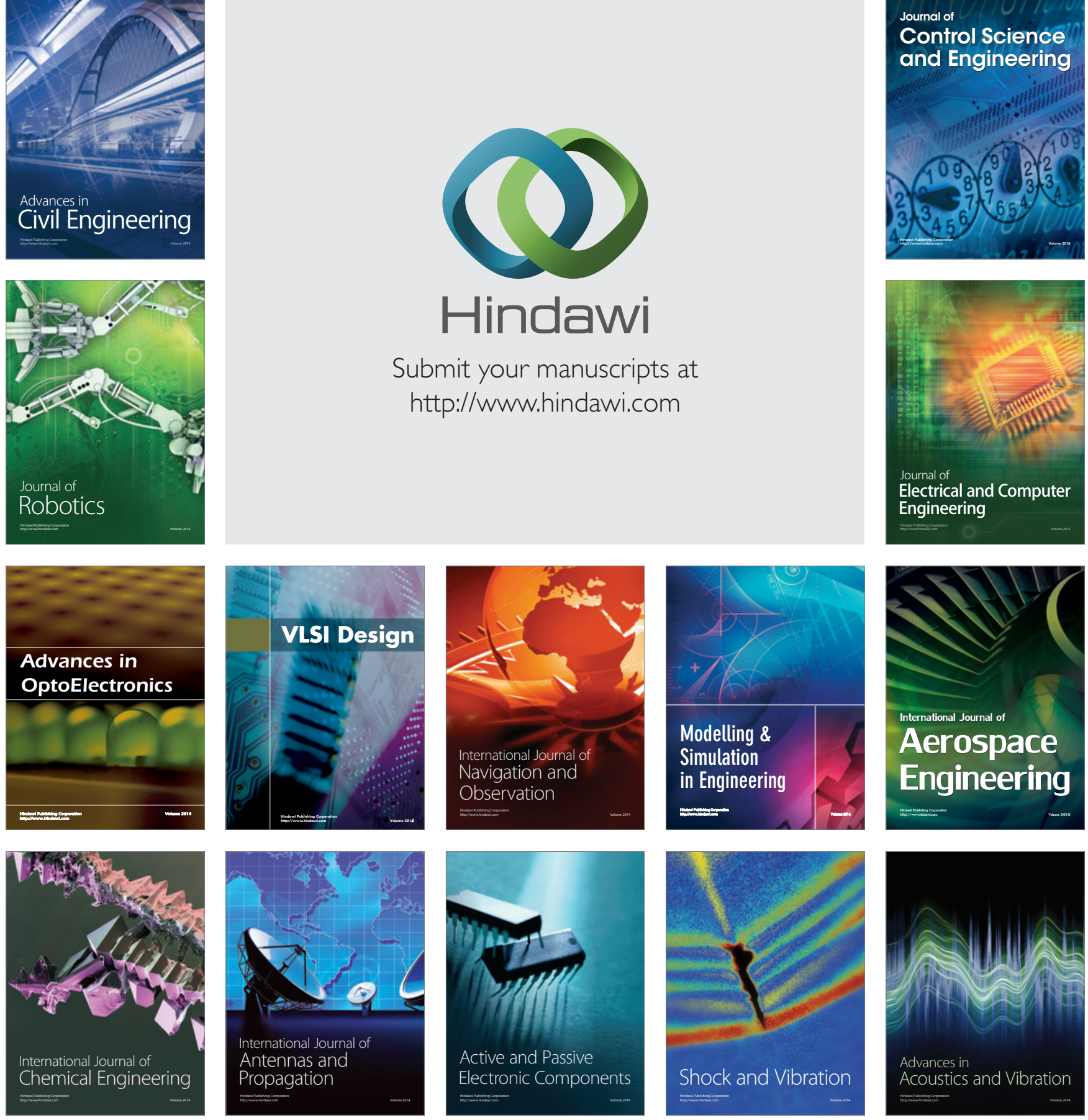